\title{
ZUR THEORIE DER GAMMAFUNCTION'
}

\author{
VON
}

\section{HJ. MELLIN}

in HULSINGFORS.

Den Ausgangspunct der nachfolgenden Untersuchungen bildet eine Funition der allgemeinen Form

$$
F(z)=e^{\mu z z} \frac{\Gamma^{h_{1}}\left(z-a_{1}\right) \Gamma^{u_{2}}\left(z-a_{2}\right) \ldots \Gamma^{\mu_{r}}\left(z-\ldots-a_{r}\right)}{\left.\Gamma^{\nu_{1}} z-b_{1}\right) \Gamma^{\nu_{z}}\left(z-b_{2}\right) \ldots \Gamma^{\nu_{k}}\left(z-b_{v}\right)}
$$

wo $\mu_{1}, \ldots, \mu_{r}, \nu_{1}, \ldots, \nu_{s}$ positive ganze Zahlen und $\alpha, a_{1}, \ldots, a_{r}$, $b_{1}, \ldots, b_{s}$ beliebige von $z$ unablängige Grössen bezeichnen.

Die Sätze, welche Herr Prym in seiner in Crelde's Journal Bd. 82 publicirten Arbeit: Zur Theorie der Gammafunction entwickelte, können als ganz specielle Fälle von denjenigen betrachtet werden, die im Folgenden bewiesen werden solien. Indem ich von dem Mrttag-Leffler'schen Satze Gebrauch mache, erhalte ich eine grosse Anzahl neuer Transcendenten, die mit der Gammafunction sehr nahe verwandt sind. Die Theorie der Gammafunction wird gleichzeitig nicht unbedeutend erweitert. Besonders bemerkenswerth ist die formale Übereinstimmung, welche dieselbe in gewissen Hinsichten mit der Theorie der linearen Differentialgleichungen erhailt. Dieser Umstand kann, wie ich bei einer anderen Gelegenheit zeigen will, dadurch erklärt werden, dass in der That auch eine nähere Beziehung zwischen den Integralen gewisser linearen Differentialgleichungen

1 Vergl. meine Abhandlung: Om en $n y$ klass af transcendenta functioner, hvilka äro nära beslägtade med gammafunktionen, Acta soc. scient. Fennicæ, Tom. XIV, XV; I 885 , I 886.

Acta mathematica. 8. Imprimé le 19 Férrier 1886. 
Hj. Mellin.

und solchen Transcendenten, von denen in Folgenten die Rede wird, stattfindet. Diese Bezichung giebt ein Satz am Ende dieser Abhandlung.

Die Function $F(z)$ genügt offenbar der Gleichung

$$
F(z+1)=\mathbf{r}(z) I(z)
$$

wo

$$
\mathbf{r}(z)=e^{\alpha} \frac{\left(z-a_{1}\right)^{\mu_{1}}\left(z-a_{2}\right)^{h_{2}} \cdots\left(z-a_{1}\right)^{\mu_{r}}}{\left(z-b_{1}\right)^{\nu_{1}}\left(z-b_{2}\right)^{\nu_{2}} \ldots\left(z-b_{s}\right)^{\nu_{s}}}
$$

ist.

Zum Ausgangspuncte unserer Untersuchungen hätten wir eine Function der noch allgemeineren Form

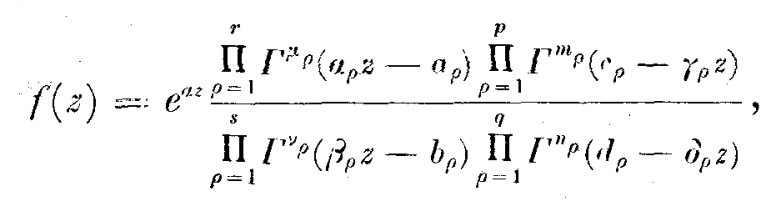

wählen können, in welcher $\mu, \nu, m, n, \alpha, \beta, \gamma, o$ positive ganze Zahlen und $a, b, c, d$ beliebige von $z$ unabhängige Grössen bezeichnen. Dass dies nicht geschehen ist, hat hauptsichlich seinen Grund in dem folgenden Satze:

Jeder belicbigen rationalew, Function $\mathbf{r}(z)$ entspricht eine und zwar, wenn man von einem Factor $e^{2 k \pi i z}$, wo $k$ eine ganze Zahl, absieht, nur eine cinzige Function, welche die Form (I) hat und der Gleichung (2) genügt. Jede andere Irunction, die derselben Gleichung genügt wie $F(z)$, kann auf die Form $\varphi(z) F(z)$ gebracht werden, wo $\varphi(z)$ eine gewisse Function mit der Periode I bezeichnet.

Auf Grund dieses Satzes, von dessen Richtigkeit man sich sofort überzeugt, können die Functionen der Form (I) als die Grundformen betrachtet werden, aus denen man alle äbrigen einer Gleichung (2) genügenden Functionen durch Multiplication mit periodischen Functionen crhailt. 


\section{1.}

Der Eigenschaft $\Gamma(z+\mathrm{I})=z \Gamma(z)$ der Gammafunction entspricht die Eigenschaft $F(z+\mathrm{I})=\mathbf{r}(z) F(z)$ yon $F(z)$. Ebenso entspricht der Eigenschaft ${ }^{1}$

$$
\lim _{m=\infty} \frac{\Gamma(z+m)}{m-\mathrm{I}} \frac{m^{z}}{\mid m}=\mathrm{I}
$$

der ersteren Function eine ähnliche Eigenschaft der letzteren. Stellt man nämlich diese Gleichung mit dem Ausdrucke von $F(z)$ zusammen, so findet man dass

$$
\lim _{m=\infty} \frac{F(z+m)}{(z, m)}=\mathbf{I}
$$

ist, wo die Grösse $(z, m)$ durch die Gleichung

$$
(z, m)=e^{a_{s}+m} \frac{\left(\mid m-\mathrm{I} m^{z-a_{1}}\right)^{\mu_{1}}\left(\mid m-\mathrm{I} m^{2-a_{2}}\right)^{\mu_{2}} \cdots\left(\mid m-\mathrm{I} m^{z-a_{r}}\right)^{a_{r}}}{\left(\mid m-\mathrm{I} m^{z-b_{1}}\right)^{\nu_{1}}\left(\mid m-\mathrm{I} m^{2-b_{2}}\right)^{\nu_{2}} \cdots\left(\mid m-\mathrm{I} m^{z-b_{i}}\right)^{\nu_{o}}}
$$

definirt ist. Setzt man zur Abkürzung

$$
\begin{gathered}
\mu=\mu_{1}+\mu_{2}+\ldots+\mu_{r}, \\
\nu=\nu_{1}+\nu_{2}+\ldots+\nu_{s}, \\
x=\nu_{1} b_{1}+\nu_{2} b_{2}+\ldots+\nu_{s} b_{s}-\mu_{1} a_{1}-\mu_{2} a_{2}-\ldots-\mu_{r} a_{r},
\end{gathered}
$$

so kann $(z, m)$ in der einfachen Form

$$
(z, m)=e^{n(z+m)}\left(\mid \underline{m-\mathrm{I}} m^{z}\right)^{u-\nu} m^{x}
$$

geschrieben werden.

$$
{ }^{1} m^{z}=\mathrm{I}+z \log m+\frac{z^{2}}{\mathrm{I} \cdot 2} \log ^{2} m+\ldots ; \text { mit } \log m \text { ist der reelle Logarithmus zu }
$$
verstehev. 
Es befriedigt also $F(z)$ ein System von Functionalgleichungen von folgender Form

$$
\left\{\begin{array}{l}
F(z+\mathrm{I})=\mathbf{r}(z) F(z) \\
\lim _{m=\infty} \frac{F(z+m)}{(z, m)}=\mathrm{I} .
\end{array}\right.
$$

Durch wiederholte Anwendung der ersteren dieser Gleichungen ergiebt sich

$F(z)=\frac{F(z+m)}{\mathbf{r}(z) \mathbf{r}(z+\mathrm{I}) \ldots \mathbf{r}(z+m-\mathfrak{I})}=\frac{F(z+m)}{(z, m)} \cdot \frac{(z, m)}{\mathbf{r}(z) \mathbf{r}(z+\mathfrak{l}) \ldots \mathbf{r}(z+m-\mathfrak{I})}$.

Wendet man auch die letztere an, so folgt

$$
F(z)=\lim _{m=\infty} \frac{e^{\alpha(z+m)}\left(\mid m-\mathrm{I} m^{2}\right)^{n-y} m^{x}}{\mathbf{r}(z) \mathbf{r}(z+\mathrm{I}) \ldots \mathbf{r}(z+m-\mathrm{I})} .
$$

Die rechte Seite dieser Gleichung hat also einen bestimmten endlichen Werth für jeden Werth $z$, der nicht gleich einer Unendlichkeitsstelle von $F(z)$ ist. Da dieser neue eindeutige Ausdruck für $F(z)$ eine nothwendige Folge der Bedingungen (8) allein ist, so geht daraus zugleich hervor, dass $F(z)$ durch diese Bedingungen eindeutig bestimmt ist.

Die Gradzahl des Zühlers von $\mathbf{r}(z)$ ist offenbar gleich $\mu$ und die des Nenners gleich $\nu$. Ist $\mu>\nu$ so ist immer

$$
\lim _{m=\infty}(z, m)=\infty
$$

ist $\mu<\nu$ so ist immer

$$
\lim _{m=\infty}(z, m)=0
$$

ist $\mu=\nu$ so ist

$$
\lim _{m=\infty}(z, m)=\infty
$$

wenn der reelle Theil von $\alpha$ positiv ist, und

$$
\lim _{m=\infty}(z, m)=0 \text {, }
$$

wenn derselbe negativ ist. Ist dagegen der reelle Theil von $\alpha$ gleich Null, so ist

$$
\lim _{m=\infty}(z, m)=\infty
$$


wenn der reelle Theil von $x$ positiv ist, und

$$
\lim _{m=\infty}(z, m)=0 \text {, }
$$

wenn derselbe negativ ist. Ist anch der reelle Theil von $\%$ gleich Null, so ist,

$$
|(z, m)|=\left|e^{a z}\right|
$$

Die letztere der Gleichungen (8) sagt also aus, wie sich die Function $F(z)$ verhält, wenn das Argument $z=x+i y$ sich dem Puncte $\infty$ in positiver Richtung längs einer der $x$-Axe parallelen Gerade nähert.

Wir setzen hier noch folgende Bezeichnungen fest, die wir künftig anwenden werden:

$$
\begin{gathered}
\mathbf{s}(z)=\frac{\mathrm{I}}{\mathbf{r}(z)} \\
\mathbf{r}_{0}(z)=e^{\prime \prime}\left(z-a_{1}\right)^{n_{1}}\left(z-a_{2}\right)^{m_{2}} \ldots\left(z-a_{r}\right)^{n_{r}} \\
\mathbf{r}_{1}(z)=\left(z-b_{1}\right)^{\nu_{1}}\left(z-b_{2}\right)^{\nu_{2}} \ldots\left(z-b_{s}\right)^{\nu_{*}} \\
\lim _{z=\infty} \mathbf{r}(z)=M .
\end{gathered}
$$

Ist die Gradzahl $\mu$ von $\mathbf{r}_{0}(z)$ grösser als die Gradzahl $\nu$ von $\mathbf{r}_{1}(z)$, so ist $M=\infty$. Ist $\mu<\nu$ so ist $M=0$. Ist $\mu=\nu$ so ist

$$
M=e^{\prime \prime}
$$

2.

Es hat sich zweckmässig gezeigt, die Constanten $a$ und $b$ in $F(z)$ einer gewissen Bedingung zu unterwerfen, und zwar der Bedingung, dass die Differenz irgend zweier dieser Grössen weder eine positive noch eine negative ganze Zahl sein darf. Vermöge der Gleichung $\Gamma(z+\mathrm{I})=z \Gamma(z)$ findet man, dass diese Bedingung nicht von einer mehr beschränkenden Natur ist, als dass $F(z)$, wenn die Bedingung nicht erfüllt wäre, auf die Form eines Productes gebracht werden könnte, dessen Factoren eine ra- 
tionale Function und eine Function derselben Form wie $F(z)$ sein würden, in der aber die Constanten $a, b$ die fragliche Bedingung erfullten.

Ist diese Bedingung festgestellt, so können niemals zwei von den $r+s$ Functionen

$$
\Gamma^{p_{1}}\left(z-a_{1}\right), \ldots, \Gamma^{\mu_{r}}\left(z-a_{v}\right), \quad \Gamma^{\nu_{1}}\left(z-b_{1}\right), \ldots, \Gamma^{\nu_{s}}\left(z-b_{s}\right)
$$

für denselben Werth $z$ unendlich werden.

Da die Gammafunction keine Nullstelle besitzt, so kann auch der Nenner von $F(z)$ für keinen Werth des Arguments gleich Null werden. Diejenigen Werthe, für die $F(z)$ unendlich wird, sind daher in den Unendlichkeitsstellen des Zühlers enthalten. Der Factor $\Gamma^{\prime \prime} p\left(z-a_{p}\right)$ des Zühlers von $F(z)$ wird immer und nur dann unendlich, wenn $z-a_{\rho}$ entweder gleich Null oder gleich einer negativen ganzen Zahl ist, d. h. für

$$
z=a_{\rho}, a_{\rho}-\mathrm{I}, \ldots, a_{p}-n, \ldots,
$$

und zwar wird er an jeder dieser Stellen unendlich der Ordnung $\mu_{p}$. Da keiner von den übrigen Factoren des Zählers und auch keiner von denen des Nenners zufolge der festgestellten Bedingung an diesen Stellen unendlich werden kann, so wird auch die Function $F(z)$ selbst für jeden der Werthe (I 4) unendlich der Ordnung $\mu_{\rho}$. Setzt man in ( I 4) $\rho=1,2, \ldots, r$, so ergeben sich sümmtliche Unendlichkeitsstellen für $F(z)$. Diese Stellen zerfallen also in $r$ arithmetische Reihen, die den $r$ Factoren im Zühler von $F(z)$ entsprechen.

In ähnlicher Weise ergiebt sich, dass die Nullstellen von $F(z)$ als Glieder in den $s$ arithmetischen Reihen enthalten sind, welche man erhält, indem man in

$$
b_{p}, b_{\rho}-\mathrm{I}, \ldots, b_{\rho}-n, \ldots
$$

den Index $\rho$ die Werthe $\mathrm{I}, 2, \ldots, s$ durchlaufen lässt. An jeder der Stellen (15) wird $F(z)$ Null von der Ordnung $\nu_{\rho}$.

Die Nullstellen des Zählers $\mathbf{r}_{\mathrm{n}}(z)$ der rationalen Function $\mathbf{r}(z)$ sind nach $\S \mathrm{I}$

$$
a_{1}, a_{2}, \ldots, a_{n}
$$

und zwar sind diese bezüglich von den Ordnungen $\mu_{1}, \mu_{2}, \ldots, \mu_{1}$. Jede 
dieser Stellen ist als erstes Glied in einer der $r$ arithmetischen Reihen enthalten, in welche die Unendlichkeitsstellen von $F(z)$ zerfallen.

Die Nullstellen des Nenners $\mathbf{r}_{1}(z)$ von $\mathbf{r}(z)$ sind

$$
b_{1}, b_{2}, \ldots, b_{s} \text {, }
$$

und zwar haben diese bezüglich die Ordnungen $\nu_{1}, \nu_{2}, \ldots, \nu_{s}$. Jede dieser Stellen ist als erstes Glied in einer der $s$ arithmetischen Reihen enthalten, in welche die Nullstellen von $F(z)$ zerfallen.

Da keine zwei von den Grössen $a, b$ einander gleich sind, so können Zähler und Nänner in $\mathbf{r}(z)$ keinen gemeinschaftlichen Theiler haben.

3.

Im ersten Theile der schon citirten Arbeit habe ich gezeigt, dass die Function $F(z)=\Gamma^{m}(z)$, welche der Gleichung

$$
F(z+\mathrm{I})=z^{\prime \prime} F(z)
$$

genügt, als Summe einer Partialbruchreihe $P(z)$ und einer bestandig convergirenden Potenzreihe $Q(z)$ dargestellt werden kann, von denen $P(z)$ einer Gleichung

$$
P(z+\mathrm{I})=z^{\prime \prime} P(z)-R(z)
$$

genügt, wo $R(z)$ eine gewisse ganze rationale Function, deren Gradzahl nicht grösser als $\mu-\mathrm{I}$ ist, bezeichnet. Hieraus folgte sodann, dass $Q(z)$ der Gleichung

Genüge leistet.

$$
Q(z+\mathrm{I})=z^{\prime \prime} Q(z)+R(z)
$$

Diese Ergebnisse geben Veranlassung zu der Vermuthung, dass sich ähnliche Sätze auch für die durch Gleichung (I) definirte allgemeinerc Function $F(z)$ beweisen lassen müssen. Diese Function befriedigt die Gleichung

$$
F(z+\mathrm{I})=\mathbf{r}(z) F(z)
$$

oder

$$
\mathbf{r}_{1}(z) F(z+1)-\mathbf{r}_{0}(z) F(z)=0
$$


Nach 'dem Mitrag-Lefrukr'schen Satze kann man

$$
F(z)=P(z)+Q(z)
$$

setzen, wo $P(z)$ eine Partialbruchreihe und $Q(z)$ eine beständig convergirende Potenzreihe bezeichnet. Es liegt nun am nächsten anzunehmen, dass $P(z)$ die Gleichung

$$
\mathbf{r}_{1}(z) P(z+1)-\mathbf{r}_{0}(z) P(z)=-R(z)
$$

befriedige, wo $R(z)$ sowie $\mathbf{r}_{0}(z)$ und $\mathbf{r}_{1}(z)$ eine ganze rationale oder wenigstens eine ganze transcendente Function bedeutet. Wäre diese Annahme richtig, so würde $Q(z)$ die Eigenschaft

$$
\mathbf{r}_{1}(z) Q(z+1)-\mathbf{r}_{0}(z) Q(z)=R(z)
$$

besitzen. Diese Gleichung sagt indessen nur in dem Falle etwas Bemerkenswerthes aus, wo sich $R(z)$ auf eine ganze rationale Function reducirt.

Die allgemeine Form der Partialbruchreihe $P(z)$ kann nach dem, was in $\$ 2$ gesagt ist, leicht aufgestellt werden. Setzt man

$$
S\left(z ; a_{\rho}\right)=\sum_{n=0}^{\infty}\left(\frac{A_{\eta_{\rho}}^{(\rho, n)}}{\left(z-a^{\rho}+n\right)^{n_{p}}}+\frac{A_{p_{\rho}-1}^{(\rho, n)}}{\left(z-a_{\rho}+n\right)^{u_{p}-1}}+\ldots+\frac{A_{1}^{(\rho, n)}}{z-a_{\rho}+n}+\mathfrak{g}_{n}\left(z, a_{\rho}\right)\right)
$$

wo $A$ Constante und $\mathfrak{g}$ ganze rationale Functionen bezeichnen, die so $z u$ wählen sind, dass die Reihe gleichmässig convergirt, so kann $P(z)$ in der Form

$$
S(z)=S\left(z ; a_{1}\right)+S\left(z ; a_{2}\right)+\ldots+S\left(z ; a_{r}\right)
$$

geschrieben werden.

Wenn die Constanten $A$ solche specielle Werthe haben, dass $S(z)$ als Partialbruchreihe für $F(z)$ betrachtet werden kann, so wenden wir folgende Bezeichnungen an

$$
\begin{array}{lll}
P(z) & \text { statt } & S(z) \\
P\left(z ; a_{p}\right) \quad » & S\left(z ; a_{p}\right) .
\end{array}
$$

Bevor wir die Constanten $A$ der Partialbruchreihe $P(z)$ bestimmen, suchen wir erst die Frage zu beantworten, ob es überhaupt möglich sei, 
in einem Ausdrucke der Form $S(z)$ die Constanten $A$ und die ganzen rationalen Functionen $g$ so zu bestimmen, dass die $r$ Reihen, von denen $S(z)$ eine Summe ist, gleichmässig convergirende Reihen ergeben und die Differenz

$$
\mathbf{r}_{1}(z) S(z+1)-\mathbf{r}_{0}(z) S(z)
$$

eine ganze, rationale oder transcendente, Function werde. Ist dies möglich, so kann die Differenz

$$
S(z+\mathbf{1})-\mathbf{r}(z) S(z)
$$

im Endlichen nur für solche Werthe unendlich werden, für welche der Nenner von $\mathbf{r}(z)$ verschwindet.

Vorausgesetzt, dass die Reihen, von denen $S(z)$ eine Summe ist, gleichmässig convergiren, so stellt der Ausdruck (20) immer und nur dann eine ganze Function dar, wenn er in der Umgebung jeder Unendlichkeitsstelle $\omega$ für $S(z)$ oder $S(z+$ I) nach positiven ganzzahligen Potenzen von $z-\omega$ entwickelt werden kann. Es müssen mithin $\mathbf{r}_{0}(z) S(z)$ und $\mathbf{r}_{1}(z) S(z+$ I) für dieselben Werthe unendlich werden und zwar so, dass in den Entwickelungen derselben nach ganzen Potenzen von $z-\omega$ die Coefficienten der gleich hohen negativen Potenzen beziehungsweise einander gleich sind.

Die Unendlichkeitsstellen von $S(z)$ sind als Glieder in den $r$ arithmetischen Reihen

$$
\begin{gathered}
a_{p}, a_{p}-\mathrm{I}, \ldots, a_{p}-n, \ldots \\
o=\mathrm{I}, 2, \ldots, r
\end{gathered}
$$

enthalten. Die ersten Glieder

$$
a_{1}, a_{2}, \ldots, a_{r}
$$

sind offenbar keine Unendlichkeitsstellen für $S(z+1)$. Das Product $\mathbf{r}_{0}(z) S(z)$ kann auch an keiner der letztgenannten Stellen unendlich werden, denn $\mathbf{r}_{0}(z)$ wird für $z=a_{\rho}$ Null von der Ordnung $\mu_{\rho}$ während $S(z)$ für denselben. Werth unendlich höchstens von der Ordnung $\mu_{p}$ werden kann. Die Differenz (20) kann mithin, auch wenn die Grössen $A$ in $S(z)$ unbestimmt gelassen werden, nur an denjenigen Stellen einen 
unendlich grossen Werth annehmen, wo $S(z)$ und $S(z+1)$ beide gleichzeitig unendlich werden. Diese für $S(z)$ und $S(z+$ I) gemeinschaftlichen Unendlichkeitsstellen sind offenbar als Glieder in den $r$ arithmetischen Reihen

$$
\begin{gathered}
a_{\rho}-1, a_{\rho}-2, \ldots, a_{\rho}-n, \ldots \\
\rho=1,2, \ldots, r
\end{gathered}
$$

enthalten, die man aus den Reihen (2 I) durch Absonderung der resp. ersten Glieder erhält. Diesen Reihen entsprechen resp. die $r$ Differenzen

$$
\begin{gathered}
\mathbf{r}_{1}(z) S\left(z+\mathrm{I} ; a_{\rho}\right)-\mathbf{r}_{0}(z) S\left(z ; a_{\rho}\right) \\
\rho=\mathrm{I}, 2, \ldots, r
\end{gathered}
$$

in welche die Differenz (20) aufgelöst werden kann und für welche sie bezüglich die Reihen der Unendlichkeitsstellen bilden, wenn die Grössen $A$ als unbestimmt betrachtet werden. Nach den Voraussetzungen, die in $\S 2$ gemacht wurden, sind je zwei dieser Reihen in den Gliedern von einander ganz verschieden, und es können also keine zwei der Differenzen (23) an derselben Stelle unendlich werden. Damit die Differenz (20) eine ganze Function sei, ist es daher nothwendig, und hinreichend, dass auch eine jede der $r$ Differenzen (23) eine ganze Function sein soll.

In der Umgebung einer für $S\left(z ; a_{\rho}\right)$ und $S\left(z+\right.$ I; $\left.a_{\rho}\right)$ gemeinschaftlichen Unendlichkeitsstelle $a_{\rho}-n, n \geqq \mathrm{I}$, hat man

$$
S\left(z ; a_{o}\right)=\frac{A_{\mu_{\rho}}^{(\rho, n)}}{\left(z-a_{\rho}+n\right)^{\mu \rho}}+\cdots+\frac{A_{1}^{(\rho, n)}}{z-a_{\rho}+n}+\mathbb{b}\left(z-a_{\rho}+n\right)
$$

und

$$
S\left(z+\mathrm{I} ; a_{\rho}\right)=\frac{A_{\mu_{\rho}}^{(\rho, n-1)}}{\left(z-a_{\rho}+n\right)^{\mu_{\rho}}}+\ldots+\frac{A_{1}^{(\rho, n-1)}}{z-a_{\rho}+n}+\left(\boldsymbol{G}_{1}\left(z-a_{\rho}+n\right),\right.
$$

wo $\mathbb{G}$ und $\sigma_{1}$ nach positiven, ganzzahligen Potenzen von $z-a_{p}+u_{\text {s }}$ fortschreitende Reihen bezeichnen. Soll nun die Differenz

$$
\mathbf{r}_{1}(z) S\left(z+\mathrm{I} ; a_{\rho}\right)-\mathbf{r}_{\mathbf{0}}(z) S\left(z ; a_{\rho}\right)
$$

eine ganze Function sein, so ist es, vorausgesetzt, dass die Reihe $S\left(z ; a_{p}\right)$ 
gleichmässig convergirt, daher nothwendig und hinreichend, die Constanten $A$ so bestimmen zu können, dass eine jede der unendlich vielen rationalen Functionen

$$
\begin{gathered}
\mathbf{r}_{1}(z)\left(\frac{A_{\mu_{p}}^{(\rho, n-1)}}{\left(z-a_{\rho}+n\right)^{n_{p}}}+\ldots+\frac{A_{1}^{(\rho, n-1)}}{z-a_{p}+n}\right) \\
-\mathbf{r}_{0}(z)\left(\frac{A_{\mu_{p}}^{(\rho, n)}}{\left(z-a_{\rho}+n\right)^{n_{p}}}+\ldots+\frac{A_{1}^{(\rho, n)}}{z-a_{o}+n}\right) \\
n=\mathrm{I}, 2,3, \ldots
\end{gathered}
$$

eine ganze rationale Function werde.

Für den reciproken Werth der rationalen Function $\mathbf{r}(z)$ haben wir die Bezeichnung $\mathbf{s}(z)$ gewählt. Da der Nenner $\mathbf{r}_{0}(z)$ von $\mathbf{s}(z)$ nach den in $\$ 2$ festgesetzten Bedingungen an keiner der Stellen

$$
z=a_{p}-n, \quad n=\mathrm{I}, 2,3, \ldots
$$

Null werden kann, so sind die Functionen (25) immer und nur dann alle ganze rationale Functionen, wenn die aus ihnen mittelst Division durch $-\mathbf{r}_{0}(z)$ abgeleiteten Functionen

$$
\begin{gathered}
\frac{A_{\mu_{p}}^{(\rho, n)}}{\left(z-a_{\rho}+n\right)^{n_{\rho}}}+\ldots+\frac{A_{1}^{(\rho, n)}}{z-a_{\rho}+n} \\
-\mathbf{s}(z)\left(\frac{A_{\mu_{\rho}}^{(\rho, n-1)}}{\left(z-a_{\rho}+n\right)^{\mu_{\rho}}}+\ldots+\frac{A_{1}^{(\rho, n+1)}}{z-a_{\rho}+n}\right) \\
n=1,2,3, \ldots
\end{gathered}
$$

bezüglich nach positiven ganzen Potenzen von

$$
z-a_{\rho}+n, \quad n=1,2,3, \ldots
$$

entwickelt werden können. Wendet man nun die Gleichung an $\mathbf{s}(z)=\mathbf{s}\left(a_{p}-n\right)+\mathbf{s}^{\prime}\left(a_{p}-n\right)\left(z-a_{p}+n\right)+\ldots+\frac{\mathbf{s}^{(k)}\left(a_{p}-n\right)}{\mid k}\left(z-a_{p}+n\right)^{k}+\ldots$, 
bewerkstelligt die Entwickelung und setzt die Coefficienten der negativen Potenzen von $z-a_{s}+n$ gleich Null, so ergeben sich die Gleichungen

$$
\begin{aligned}
& A_{\eta^{\prime}}^{(n)}=\mathbf{s}(a-n) A_{m^{\prime}}^{(n-1)} \\
& A_{n^{\prime}-1}^{(n)}=-\mathbf{s}^{\prime}(a-n) A_{n^{\prime}}^{(n-1)}+\mathbf{s}(a-n) A_{n^{\prime}-1}^{(n-1)} \\
& \text { (26) } A_{k^{\prime}-l}^{(n)}=\frac{\mathbf{s}^{(k)}(a-n)}{\mid \underline{k}} A_{\eta^{\prime}}^{(n-1)}+\frac{\mathbf{s}^{(k-1)}(a-n)}{\mid \underline{k-1}} A_{n^{\prime}-1}^{(n-1)}+\ldots+\mathbf{s}(n-n) A_{u^{\prime}-k}^{(n-1)} \\
& A_{1}^{(n)}=\frac{\mathbf{s}^{\left(\mu^{\prime}-1\right)}(a-n)}{\underline{\mu^{\prime}-1}} A_{n^{\prime}}^{(n-1)}+\frac{\mathbf{s}^{\left(\mu^{\prime}-2\right)}(a-n)}{\mid \underline{\mu^{\prime}-2}} A_{\eta^{\prime}-1}^{(n-1)}+\ldots+\mathbf{s}(a-n) A_{1}^{(n-1)}, \\
& n=\mathrm{I}, 2,3, \ldots,
\end{aligned}
$$

wo der Index $\rho$ der Kürze halber überall fortgelassen ist, indem ich

gesetzt habe.

$$
A_{\mu_{0}, k}^{(o, n)}=A_{\mu^{\prime}-k,}^{(n)}, \quad a_{\rho}=a, \quad \mu_{o}=\mu^{\prime}
$$

Unter Voraussetzung der gleichmässigen Convergenz der Reihe $S\left(z ; a_{\rho}\right)$ drucken die recurrirenden Gleichungen (26) die nothwendige und hinreichende Bedingung dafür aus, dass die Differenz (24) eine ganze Function sein soll.

Sobald den Constanten

$$
A_{\eta^{\prime}}^{(0)}, A_{p^{\prime}-1}^{(0)}, \ldots, A_{1}^{(0)}
$$

des ersten Gliedes von $S\left(z ; a_{n}\right)$ beliebige bestimmte Werthe znertheilt werden, so sind die Constanten $A$ der folgenden Glieder durch die Formeln (26) eindeutig bestimmt, und zwar sind für jedes $n$

$$
A_{\mu^{\prime}}^{(n)}, A_{r^{\prime}-1}^{(n)}, \ldots, A_{i}^{(n)}
$$

homogene lineare Functionen von

$$
A_{i^{\prime}}^{(0)}, A_{r^{\prime}-1}^{(0)}, \ldots, A_{1}^{(0)} \text {. }
$$

Bedenkt man nun, dass man immer, wie auch die Constanten $A$ des ersten Gliedes von $S\left(z ; a_{o}\right)$ gewählt worden seien, nachdem den- 
jenigen der folgenden Glieder die aus den Formeln (26) sich ergebenden Werthe zuertheilt worden sind, solche ganze rationale Functionen $\mathfrak{g}_{0}\left(z ; a_{\rho}\right)$, $\mathfrak{g}_{1}\left(z ; a_{p}\right), \ldots$ darstellen kann, dass $S\left(z ; a_{p}\right)$ zu einer gleichmässig convergirenden Reihe wird, so geht hieraus hervor, dass es unendlich viele Functionen der Form $S\left(z ; a_{\rho}\right)$ geben muss, welche die Eigenschaft besitzen, die Gleichung

$$
\mathbf{r}_{1}(z) S\left(z+\mathrm{I} ; a_{\rho}\right)=\mathbf{r}_{0}(z) S\left(z ; a_{\rho}\right)-R\left(z ; a_{\rho}\right)
$$

zu befriedigen, wo $R\left(z ; a_{\rho}\right)$ den Charakter einer ganzen Function hat. Wird auf beide Seiten dieser Gleichung durch $\mathbf{r}_{1}(z)$ dividirt, so folgt

$$
S\left(z+\mathrm{I} ; a_{\rho}\right)=\mathbf{r}(z) S\left(z ; a_{\rho}\right)-\mathbf{R}\left(z ; a_{\rho}\right)
$$

wo

$$
\mathbf{R}\left(z ; a_{\rho}\right)=\frac{R\left(z ; a_{\rho}\right)}{\mathbf{r}_{1}(z)}
$$

Dic Gleichung $(27)$ drückt eine bemerkenswerthe Eigenschaft der Function $S\left(z ; a_{\rho}\right)$ aus, weil die beiden Functionen $\mathbf{r}(z)$ und $\mathbf{R}\left(z ; a_{\rho}\right)$ nur eine endliche Anzahl von Únendlichkeitsstellen haben, während die Anzahl derselben für $S\left(z ; a_{\rho}\right)$ eine unendliche ist.

Auf Grund der jetzt gewonnenen Resultate ergiebt sich in Bezug auf den Ausdruck $S(z)$, dessen allgemeine Form die Gleichungen (1 8 ) und (19) angeben, Folgendes. Es giebt unendlich viele Functionen dieser Form, welche die Eigenschaft besitzen, der Gleichung

$$
S(z+\mathrm{I})=\mathbf{r}(z) S(z)-\mathbf{R}(z)
$$

zu genügen, wo $\mathbf{R}(z)$ eine Function bezeichnet, welche in der Form eines Qvotienten, dessen Zähler eine ganze Function und dessen Nenner die rationale ganze Function $\mathbf{r}_{1}(z)$ ist, dargestellt werden kann. Soll $S(z)$ dieser Gleichung genügen, so müssen vor allem die Constanten $A$ der Reihen, von denen $S(z)$ eine Surnme ist, durch $r$ diesen Reihen entsprechende Gleichungssysteme der Form (26) bestimmt werden. Sind ausserdem die ganzen rationalen Functionen $\mathfrak{g}$ so bestimmt, dass diese $r$ Reihen gleichmässig convergiren, so sind zugleich alle Bedingungen dafür, dass $S(z)$ eine Function der angegebenen Beschaffenheit darstelle, erfüllt. 
Hj. Mellin.

Die zu $F(z)$ gehörige Partialbruchreihe $P(z)$ hat dieselbe Form wie $S(z)$. Es ist nun die Frage $z u$ beantworten, ob sie auch einer Gleichung derselben Form genügt wie $S(z)$.

4.

Wir nehmen an, dass die Constanten $A$ in dem allgemeinen Ausdrucke $S(z)$ solche specielle Werthe haben, dass wir

$$
\begin{gathered}
S(z)=P(z) \\
S\left(z ; a_{\rho}\right)=P\left(z ; a_{\rho}\right) \\
\rho=\mathrm{I}, 2, \ldots, r
\end{gathered}
$$

setzen können, und werden zeigen, dass die zur Reihe $P\left(z ; a_{o}\right)$ gehörigen Constanten $A$ die Bedingungen (26) erfüllen. Damit ist auch bewiesen, dass $P\left(z ; a_{\rho}\right)$ die Gleichung

$$
P\left(z+\mathrm{I} ; a_{\rho}\right)=\mathbf{r}(z) P\left(z ; a_{\rho}\right)-\mathbf{R}\left(z ; a_{\rho}\right)
$$

und mithin $P(z)$ die Gleichung

$$
P(z+\mathrm{I})=\mathbf{r}(z) P(z)-\mathbf{R}(z)
$$

erfüllt, wo mit $\mathbf{R}\left(z ; a_{o}\right)$ und $\mathbf{R}(z)$ Functionen bezeichnet werden, die sich beide in der Form eines Qvotienten, dessen Zähler eine ganze Function und dessen Nenner die rationale ganze Function $\mathbf{r}_{1}(z)$ ist, darstellen lassen.

Wenn die Constanten $A$ die fraglichen Werthe besitzen, so gilt für eine gewisse Umgebung der Stelle

die Gleichung

$$
z=a_{\rho}-n
$$

$$
F(z)=\frac{A_{n^{\prime}}^{(n)}}{(z-a+n)^{\prime^{\prime}}}+\ldots+\frac{A_{1}^{(n)}}{z-a+n}+\mathbb{G}(z-a+n),
$$

wo

$$
A_{\mu^{\prime}-k}^{(n)}=A_{\mu_{p}-k}^{(\rho, m)}, \quad a=a_{p}, \quad \mu^{\prime}=\mu_{\rho}
$$


und 6 eine nach positiven ganzzahligen Potenzen von $z-a+n$ fortschreitende Reihe bezeichnet. Bekanntlich kann man nun eine jede der Grössen $A$ in der Form eines bestimmten Integrals ausdrücken, und zwar ist

$$
A_{n^{\prime}-k}^{(n)}=\frac{\mathrm{I}}{2 \pi} \int_{0}^{2 \pi} F\left(a-n+r e^{i t}\right)\left(r e^{i t}\right)^{\mu^{\prime}-k} d t
$$

wo $r$ eine beliebige positive Grösse, welche kleiner als der Convergenzradius der Reihe $\mathbb{G}$ ist, bezeichnet. Aus (29) ergiebt sich

$$
A_{i^{\prime}-k}^{(n-1)}=\frac{\mathrm{I}}{2 \pi} \int_{0}^{2 \pi} F\left(a-n+1+r e^{i l}\right)\left(r e^{i t}\right)^{\mu^{\prime}-k} d t
$$

Setzt man in (29)

$$
\begin{aligned}
F\left(a-n+r e^{i t}\right) & =F\left(a-n+\mathrm{I}+r e^{i t}\right) \mathbf{s}\left(a-n+r e^{i t}\right) \\
& =F\left(a-n+\mathrm{I}+r e^{i t}\right)\left(\sum_{\lambda=0}^{i=k} \frac{\mathbf{s}^{(\lambda)}(a-n)}{\mid \underline{\lambda}}\left(r e^{i t}\right)^{\lambda}+\left(r e^{i t}\right)^{k+1} f\left(r e^{i t}\right)\right),
\end{aligned}
$$

führt dic Integration gliedweisc aus und beachtet die Gleichung (30), so folgt

$$
\begin{aligned}
A_{\mu^{\prime}-k}^{(n)}= & \mathbf{s}(a-n) A_{p^{\prime}-k}^{(n-1)}+\mathbf{s}^{\prime}(a-n) A_{\mu^{\prime}-k+1}^{(n-1)}+\ldots+\frac{\mathbf{s}^{(k)}(a-n)}{\mid k} A_{p^{\prime}}^{(n-1)} \\
& +\frac{\mathrm{I}}{2 \pi} \int_{0}^{2 \pi} F\left(a-n+\mathrm{I}+r e^{i t}\right)\left(r e^{i t}\right)^{n^{\prime}+1} f\left(r e^{i t}\right) d t .
\end{aligned}
$$

Hier bezeichnet $f\left(r e^{i t}\right)$ offenbar eine in Bezug auf $r e^{i t}$ rationale Function, die für $r=0$ einen endlichen Werth annimmt. Weil

$$
\lim _{r=0} F\left(a-n+\mathrm{I}+r e^{i t}\right)\left(r e^{i t}\right)^{\mu^{\prime}}
$$

eine endliche Grösse ist, und mithin

$$
\lim _{r=0} F\left(a-n+\mathrm{I}+r e^{i t}\right)\left(r e^{i t}\right)^{a+1} f\left(r e^{i t}\right)=0,
$$

so ist das letrte Glied der rechten Scite von (31) ebenfalls gleich Null, 
Wird dasselbe aus der Gleichung fortgelassen und werden die übrigen Glieder der rechten Seite in umgekehrter Reihenfolge geschrieben, so erhält man

$$
\dot{A_{\mu^{\prime}-k}^{(n)}}=\frac{\mathbf{s}^{(k)}(a-n)}{\mid \underline{k}} A_{\mu^{\prime}}^{(n-1)}+\frac{\mathbf{s}^{(k-1)}(a-n)}{\mid \underline{k-1}} A_{\mu^{\prime}-k}^{(n-1)}+\ldots+\mathbf{s}(a-n) A_{n^{\prime}-k}^{(n-1)} .
$$

Diese Gleichung zeigt, dass die Constanten $A$ der Partialbruchreihe $P\left(z ; a_{\rho}\right)$ den Bedingungen (26) genügen.

Es ist mithin eine ganz allgemeine und zugleich bemerkenswerthe Eigenschaft der Partialbruchreihe $P(z)$ jeder Function $F(z)$, welche die in $\S 2$ erwähnten Bedingungen erfüllt, dass sie der Gleichung

$$
P(z+\mathrm{I})=\mathbf{r}(z) P(z)-\mathbf{R}(z)
$$

genügt, mithin eine ähnliche Eigenschaft besitat wie die Function $F(z)$, welche die Gleichung

$$
F(z+\mathrm{I})=\mathbf{r}(z) F(z)
$$

befriedigt. Die Functic $n \mathbf{R}(z)$ ist in der Form eines Qvotienten darstellbar, dessen Zühler eine ganze Function und dessen Nenner gleich demjenigen von $\mathbf{r}(z)$ ist.

Aus den vorigen Untersuchungen hat sich ferner ergeben, dass es unendlich viele andere Functionen giebt, welche gleichwie $P(z)$ einer Gleichung der Form (28) genügen.

Unter diesen Functionen $S(z)$, welche die Eigenschaft besitzen, einer Gleichung der Form (28) zu genügen, sind natürlich diejenigen am meisten bemerkenswerth, für welche $\mathbf{R}(z)$ sich auf eine rationale Function reducirt.

Bis jetzt ist die rationale Function $\mathbf{r}(z)$ keinen anderen Bedingungen unterworfen gewesen, als dass der Zähler keine Constante und die Differenz irgend zweier der $r+s$ Grössen $a, b$ keine ganze Zahl sei. Ist $\mathbf{r}(z)$ gegeben, so ist auch diejenige Function der Form (I), die der Gleichung

$$
F(z+\mathrm{I})=\mathbf{r}(z) F(z)
$$

genügt, eindeutig bestimmt bis auf einen Factor $e^{2 k \pi i z}$, wo $k$ eine ganze Zahl. Umgekehrt ist $\mathbf{r}(z)$ eindeutig bestimmt, wenn $F(z)$ gegeben ist. 
Weiterhin werden wir berücksichtigen ob, die Grösse

$$
\lim _{z=\infty} \mathbf{r}(z)=M
$$

dem absoluten Betrage nach grösser als, gleich oder kleiner als I ist. Von nun an soll vorausgesetzt sein, dass

$$
|M|>\mathrm{I}
$$

Für diesen Fall wollen wir zunächst zeigen, dass die Reihen

$$
S\left(z ; a_{1}\right), \quad S\left(z ; a_{2}\right), \ldots . \quad, \quad S\left(z ; a_{r}\right),
$$

von denen $S(z)$ eine Summe ist, auch dann gleichmässig convergiren, wenn man die in den Gliedern derselben vorkommenden ganzen rationalen Functionen g gleich Null annimmt. Alle auf diese Weise crhaltenen Functionen $S(z)$ zeichnen sich dadurch aus, dass die entsprechenden Functionen $\mathbf{R}(z)$ immer rational sind.

Von grossem Interesse ist der Fall, wo $|M|=\mathrm{I}$ ist; hierher gehören beispielsweise die Potenzen des EuLur'schen Integrals erster Gattung

$$
\int_{0}^{1} t^{z-1}(1-t)^{a-1} d t=\frac{\Gamma(z) \Gamma(a)}{\Gamma^{\prime}(z+a)} .
$$

In diesem Falle convergiren die Reihen $S(z)$ zwar nicht immer gleichmäsig, wenn die ganzen rationalen Functionen g gleich Null angenommen werden, wohl aber kann man bewirken, dass die Reihen gleichmässig convergiren, ohne dass die Gradzahl der Functionen g mit der Ordnungszahl über jede Grenze hinaus zu wachsen braucht. Die Untersuchung für den Fall, wo $|M|=$ I ist, will ich einer späteren Arbeit aufbewahren, da sie nicht durch so einfache Betrachtungen, wie sie weiterhin in Frage kommen, erledigt werden kann.

Von nicht so grosse Interesse scheint der Fall zu sein, wo $|M|<\mathrm{I}$ ist, weil die Gradzahl der Functionen $\mathbf{g}$ mit der Ordnungszahl über jede Grenze hieraus wachsen muss, damit die Reihen $S(z)$ gleichmässig convergiren. 
5.

Wir setzen also im Folgenden voraus, dass die Gradzahl des Zählers von $\mathbf{r}(z)$ entweder grösser als die des Nenners oder gleich derselben sei, und, wenn das letztere zutrifft, dass

$$
\lim _{z=\infty} \mathbf{r}(z)=M
$$

dem absoluten Betrage nach grösser als $I$ ist, mit andern Worten, dass

$$
0 \leqq\left|\frac{I}{M}\right|<\mathrm{I} .
$$

Ist dann

$$
\begin{aligned}
\text { (32) } S\left(z ; a_{\rho}\right)=\sum_{n=0}^{\infty} & \left(\frac{A_{\mu^{\prime}}^{(n)}}{(z-a+n)^{n^{\prime}}}+\frac{A_{\mu^{\prime}-1}^{(n)}}{(z-a+n)^{z^{\prime}-1}}+\cdots+\frac{A_{1}^{(n)}}{z-a+n}\right) \\
& \left(A_{\mu^{\prime}-k}^{(n)}=A_{\mu_{\rho}-k}^{(\rho, n)}, a=a_{\rho}, \mu^{\prime}=\mu_{\rho}\right)
\end{aligned}
$$

eine Partialbruchreihe, in der die Grössen $A$ die Bedingungen (26) erfüllen, so kann bewiesen werden, dass diese Reihe gleichmässig convergirt. - Es sei nämlich $\varepsilon$ eine positive Grösse, welche die Bedingung

$$
\left|\frac{\mathrm{I}}{M}\right|<\varepsilon<\mathrm{I}
$$

erfüllt. Weil

$$
\mathbf{s}(z)=\frac{\mathrm{I}}{\mathbf{r}(z)}
$$

ist, und mithin

$$
\lim _{z=\infty} \mathbf{s}(z)=\frac{\mathrm{I}}{M},
$$

so kann immer eine positive Grösse $\rho$ so angenommen werden, dass

$$
|\mathbf{s}(z)|<\varepsilon
$$

wenn die Veränderliche $z$ dem absoluten Betrage nach grösser als $\rho$ ist. Wird $\mathbf{s}(\tilde{z})$ in der bekannten Form einer Summe von Partialbrüchen und 
einer ganzen Function dargestellt, so muss offenbar diese ganze Function gleich $\frac{\mathrm{I}}{M}$ sein. Werden nun durch Differentiation ähnliche Ausdrücke für die Ableitungen von $\mathbf{s}(z)$ entwickelt, so leuchtet unmittelbar ein, dass

$$
\lim _{z=\infty} \mathbf{s}^{(k)}(z)=0, \quad k=\mathrm{I}, 2,3, \ldots
$$

Bezeichnet also $\partial$ eine positive Grösse, welche die Bedingung

$$
\varepsilon+\left(\mu^{\prime}-\mathrm{I}\right) \grave{j}<\mathrm{I}, \quad \mu^{\prime}=\mu_{\rho}
$$

erfüllt, so ist es immer möglich, eine positive ganze Zahl $n^{\prime}$ so anzunehmen, dass die Ungleichheiten

$$
|\mathbf{s}(a-n)|<\varepsilon,\left|\mathbf{s}^{\prime}(a-n)\right|<o, \ldots,\left|\mathbf{s}^{\left(x^{\prime}-1\right)}(a-n)\right|<o
$$

stattfinden, wenn $n$ grösser als $n^{\prime}$ ist. Aus den Gleichungen (26) geh1 nun hervor, dass

$$
\begin{aligned}
& \left|A_{k^{\prime}}^{(n)}\right| \leqq \S\left|A_{\mu^{\prime}}^{(n-1)}\right| \\
& \left|A_{p^{\prime}-1}^{(n)}\right| \leqq \partial\left|A_{n^{\prime}}^{(n-1)}\right|+\varepsilon\left|A_{n^{\prime}-1}^{(n-1)}\right| \\
& \left|A_{n^{\prime}-2}^{(n)}\right| \leq \partial\left|A_{\eta^{\prime}}^{(n-1)}\right|+\partial\left|A_{n^{\prime}-1}^{(n-1)}\right|+\varepsilon\left|A_{n^{i}-2}^{(n-1)}\right|
\end{aligned}
$$

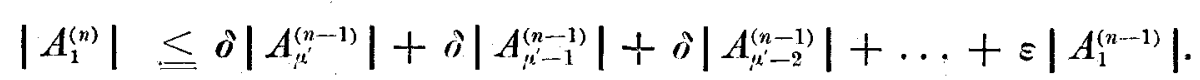

Es ist also

$$
\begin{gathered}
\left|A_{n^{\prime}}^{(n)}\right|+\left|A_{n^{\prime}-1}^{(n)}\right|+\ldots+\left|A_{1}^{(n)}\right| \\
\leqq\left[\varepsilon+\left(\mu^{\prime}-\mathrm{I}\right) \delta\right]\left|A_{n^{\prime}}^{(n-1)}\right|+\left[\varepsilon+\left(\mu^{\prime}-2\right) \delta\right]\left|A_{p^{\prime}-1}^{(n-1)}\right|+\ldots+\varepsilon\left|A_{1}^{(n-1)}\right|
\end{gathered}
$$

und mithin

$$
\begin{gathered}
\left|A_{\mu^{\prime}}^{(n)}\right|+\left|A_{k^{\prime}-1}^{(n)}\right|+\ldots+\left|A_{1}^{(n)}\right| \\
\leq\left[\varepsilon+\left(\mu^{\prime}-\mathrm{I}\right) \rho\right]\left(\left|A_{\mu^{\prime}}^{(n-1)}\right|+\left|A_{\mu^{\prime}-1}^{(n-1)}\right|+\ldots+\left|A_{1}^{(n-1)}\right|\right) .
\end{gathered}
$$

Weil $\varepsilon+\left(\mu^{\prime}-\mathrm{I}\right) \partial<\mathrm{I}$ so ist

$$
\sum_{n=0}^{\infty}\left(\left|A_{\mu^{\prime}}^{(n)}\right|+\left|A_{n^{\prime}-1}^{(n)}\right|+\ldots+\left|A_{1}^{(n)}\right|\right)
$$


Hj. Mellin.

eine convergirende Reihe. Nachdem nun die Convergenz der Reihe (33) nachgewiesen ist, so ergeben die gewöhnlichen Schlussfolgerungen, dass die Reihe (32) eine unbedingt und gleichmässig convergirende ist.

Mit der Bezeichnung $S\left(z ; a_{p}\right)$ soll im weiteren Verlaufe dieser Untersuchungen immer eine Reihe verstanden sein, welche durch die Gleichung (32) definirt ist. Die Constanten $A$ sind den Bedingungen (26) unterworfen, durch welche die Constanten

$$
A_{p^{\prime}}^{(0)}, A_{p^{\prime}-1}^{(0)}, \ldots, A_{1}^{(0)}
$$

des ersten Gliedes von $S\left(z ; a_{p}\right)$ nicht bestimmt werden. Indem man diesen Constanten verschiedene Werthe beilegt, erhalt man eine unendliche Anzahl von Functionen, von denen jede einer Gleichung

$$
S\left(z+\mathrm{I} ; a_{\rho}\right)=\mathbf{r}(z) S\left(z ; a_{\rho}\right)-\mathbf{R}\left(z ; a_{o}\right)
$$

Genüge leistet.

Legt man ferner dem Index $\rho$ in der Gleichung (32), wo der Kürze halber

$$
A_{\mu^{2}{ }^{\prime}-k}^{(0, n)}=A_{\mu^{\prime}}^{(n)}, \quad a_{\rho}=a, \quad \mu_{\rho}=\mu^{\prime}
$$

gesetzt sind, die Werthe $\rho=\mathrm{I}, 2,3, \ldots, r$ bei, so entstehen $r$ verschiedene Gruppen von Functionen. $\mathrm{Zu}$ jeder dieser Gruppen gehört ein System von recurrirenden Gleichungen der Form (26), mittelst dessen die Constanten $A$ jeder zur Gruppe gehörigen Function berechnet werden können. Dieses System ist von einem zu einer anderen Gruppe gehörigen Systeme wenigstens in den Coefficienten verschieden. Die Reihen

$$
P\left(z ; a_{1}\right), \quad P\left(z ; a_{2}\right), \quad . \quad . \quad P\left(z ; a_{r}\right)
$$

deren Summe

$$
P(z)=P\left(z ; a_{1}\right)+P\left(z ; a_{2}\right)+\ldots+P\left(z ; a_{r}\right)
$$

gleich der zu $F(z)$ gehörigen Partialbruchreihe ist, gehören zu je einer dieser $r$ Gruppen. Diejenige Gruppe, zu welcher $P\left(z ; a_{g}\right)$ gehört, wollen wir kurz mit $\left(a_{p}\right)$ bezeichnen. Nach den in $\$ 2$ erwähnten Bedingungen können zwei zu verschiedenen Gruppen gehörige Reihen für denselben Werth der Veränderlichen z nicht unendlich werden. Dagegen werden 
Zur Theorie der Gammafunction.

alle zur Gruppe $\left(a_{\rho}\right)$ gehörigen Reihen unendlich, wenn die Veränderliche $z$ mit einem Gliede der arithmetischen Reihe

$$
a_{\rho}, a_{\rho}-\mathrm{I}, \ldots, a_{\rho}-n, \ldots
$$

zusammenfällt.

Die Richtigkeit dieser letzten Behauptung geht aus dem Satze hervor, dass die Constanten $A$ desselben Gliedes einer Reihe $S\left(z ; a_{\rho}\right)$ nicht gleichzeitig Null sein können, wenn nicht $S\left(z ; a_{\rho}\right)$ identisch gleich Null sein soll. Dieses folgt aus den Gleichungen (26) und zwar, weil nicht nur eine jede der Constanten

$$
A_{\mu^{\prime}}^{(n)}, A_{\mu^{\prime}-1}^{(n)}, \ldots, A_{1}^{(n)}
$$

als eine homogene lineare Function von

$$
A_{\mu^{\prime}}^{(0)}, A_{\mu^{\prime}-1}^{(0)}, \ldots, A_{\mathrm{i}}^{(0)}
$$

ausgedrückt werden kann, sondern auch eine jede der letzteren als homogene lineare Function der ersteren, da $\mathbf{s}(a-n)$ von Null verschieden ist.

Aus den nämlichen Gleichungen leuchtet auch die Richtigkeit des folgenden Satzes ein: Wenn jede der Constanten $A$ des ersten Gliedes einer zur Gruppe $\left(a_{\rho}\right)$ gehörigen Reihe gleich der entsprechenden Constante einer anderen zu derselben Gruppe gehörigen Reihe ist, so sind die beiden Reihen idenisch.

Ferner kann offenbar jede homogene und lineare Function einer Anzahl zu derselben Gruppe gehöriger Reihen gleich einer einzigen zur Gruppe gehörigen Reihe gesetzt werden.

Es seien

$$
S_{1}\left(z ; a_{\rho}\right), \quad S_{2}\left(z ; a_{\rho}\right), \quad \cdot \quad \cdot \quad S_{\mu_{\rho}}\left(z ; a_{\rho}\right)
$$

$\mu_{o}$ zur Gruppe $\left(a_{o}\right)$ gehörige Reihen und

$$
\begin{aligned}
& A_{11}^{(\rho)}, A_{12}^{(\rho)}, \ldots, A_{\mu_{\mu} \rho}^{(\rho)} \\
& A_{21}^{(\rho)}, A_{22}^{(\rho)}, \ldots, A_{2_{\mu}^{\prime} \rho}^{(\rho)} \\
& \cdot \cdot \cdot \cdot, \cdot \cdot \cdot \\
& A_{\mu_{\rho} 1}^{(\rho)}, A_{\mu_{\rho} 2}^{(\rho)}, \ldots, A_{\mu_{\rho} \mu_{\rho}}^{(\rho)}
\end{aligned}
$$


die zu den bezüglichen ersten Gliedern derselben gehörigen Constanten. Sind nun die Reihen $S$ so gewühlt, dass die Determinante

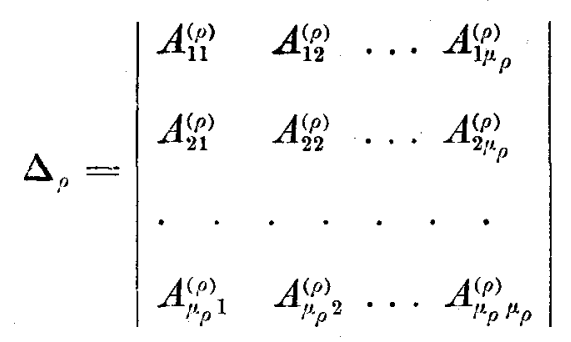

von Null verschieden ist, so kann jede andere zur Gruppe $\left(a_{\rho}\right)$ gehörige Reihe $S\left(z ; a_{\rho}\right)$ immer und nur auf eine Weise als homogene und lineare Function von $S_{1}, S_{2}, \ldots, S_{\mu_{p}}$ ausgedrückt werden, d. h. in der Form

$$
S\left(z ; a_{\rho}\right)=p_{1}^{(o)} S_{1}\left(z ; a_{\rho}\right)+p_{2}^{(\rho)} S_{2}\left(z ; a_{\rho}\right)+\ldots+p_{\eta_{\rho}}^{(\rho)} S_{n_{\rho}}\left(z ; a_{\rho}\right) \text {. }
$$

Denn, falls

$$
A_{1}^{(\rho)}, A_{2}^{(\rho)}, \ldots, A_{\mu_{\rho}}^{(\rho)}
$$

die Constanten des ersten Gliedes von $S\left(z ; a_{p}\right)$ bezeichnen, so giebt es, weil die Determinante $\Delta_{\rho}$ des linearen Gleichungssystems

$$
\begin{aligned}
& A_{11}^{(\rho)} p_{1}^{(\rho)}+A_{21}^{(\rho)} p_{2}^{(\rho)}+\ldots+A_{\mu^{\prime} \rho^{1}}^{(\rho)} p_{\nu_{\rho}}^{(\rho)}=A_{1}^{(\rho)} \\
& A_{12}^{(\rho)} p_{1}^{(\rho)}+A_{22}^{(\rho)} p_{2}^{(\rho)}+\ldots+A_{\mu_{\rho} 2}^{(\rho)} p_{\mu_{\rho}}^{(\rho)}=A_{2}^{(\rho)} \\
& A_{1_{\mu} \rho}^{(\rho)} p_{1}^{(\rho)}+A_{2_{i \mu_{\rho}}}^{(\rho)} p_{2}^{(\rho)}+\ldots+A_{\mu_{\rho} \mu_{\beta} \mu_{\mu_{\rho}}^{(\rho)}}^{(\rho)}=A_{\mu_{\rho}}^{(\mu)}
\end{aligned}
$$

unserer Annahme nach von Null verschieden ist, ein, und zwar nur ein einziges, System

$$
p_{1}^{(\rho)}, p_{2}^{(\rho)}, \ldots, p_{\mu_{\rho}}^{(\rho)}
$$

welches dem Gleichungssysteme genügt, und für welches auf Grund der beiden vorhergehenden Sätze die Gleichung (36) besteht.

Betrachten wir jetzt die Functionen, welche durch den allgemeinen Ausdruck

$$
S(z)=\sum_{\rho=1}^{r} S\left(z ; a_{\rho}\right)
$$


darrgestellt werden, wo $S\left(z ; a_{\rho}\right)$ eine beliebige zur Gruppe $\left(a_{\rho}\right)$ gehörige Function bezeichnet, so ist

$$
S(z+\mathrm{I})=\mathbf{r}(z) S(z)-\mathbf{R}(z)
$$

und

$$
\mathbf{R}(z)=\sum_{\rho=1}^{r} \mathbf{R}\left(z ; a_{\rho}\right)
$$

Es seien

$$
S_{1}\left(z ; a_{\rho}\right), \quad S_{2}\left(z ; a_{\rho}\right), \quad . \quad . \quad, \quad S_{\mu_{\rho}}\left(z ; a_{\rho}\right)
$$

wie vorher $\mu_{\rho}$ zur Gruppe $\left(a_{\rho}\right)$ gehörige Reihen, die so gewählt sind, dass die Determinante $\Delta_{p}$ einen von Null verschiedenen Werth hat. Es besteht dann die Gleichung (36), mit deren Hülfe $S(z)$ auf die Form

$$
S(z)=\sum_{\rho=1}^{r}\left[p_{1}^{(\rho)} S_{1}\left(z ; a_{\rho}\right)+p_{2}^{(\rho)} S_{2}\left(z ; a_{\rho}\right)+\ldots+p_{\mu_{\rho}}^{(\rho)} S_{\mu_{\rho}}\left(z ; a_{\rho}\right)\right]
$$

gebracht werden kann. In diesem Ausdrucke kominen $\mu_{1}+\mu_{2}+\ldots+\mu_{r}=\mu$ Reihen vor und ebenso viele Constanten $p$. Dadurch, dass man nur diese Constanten verändert, werden alle Functionen erhalten, die durch den Ausdruck (38) dargestellt werden. Sind die indicirten Reihen gegeben und handelt es sich darum, $S(z)$ in der Form (4I) darzustellen, so hat man die Constanten $p$ durch ein System von $\mu_{1}+\mu_{2}+\ldots+\mu_{\rho}=\mu$ linearen Gleichungen zu bestimmen, wodurch die $r$ Systeme, welche man dadurch erhält, dass man in (37) nach einander $\rho=1,2, \ldots, r$ setzt, zusammenbegriffen werden. Wird die Determinante des so erhaltenen Systems mit $\Delta$ bezeichnet, so ist

$$
\Delta=\left|\begin{array}{llll}
\bar{\Delta}_{1} & & & \\
& \bar{\Delta}_{2} & & \\
& & \ddots & \\
& & & \bar{\Delta}_{r}
\end{array}\right|
$$


wenn $\bar{\Delta}_{p}$ das Schema

$$
\begin{aligned}
& A_{11}^{(\rho)} \ldots A_{\mu_{\rho} 1}^{(\rho)} \\
& \ldots \ldots \\
& A_{\mu_{\rho} 1}^{(\rho)} \ldots A_{\mu_{\rho} \mu_{\rho}}^{(\rho)}
\end{aligned}
$$

bezeichnet. Aus dieser Form der Determinante $\Delta$ geht hervor, dass

$$
\Delta=\Delta_{1} \Delta_{2} \ldots \Delta_{r} .
$$

6.

Wir wollen nun nachweisen, dass in der Gleichung (34), welcher eine zur Gruppe $\left(a_{\rho}\right)$ gehörige Reihe genügt, die Function $\mathbf{R}\left(z ; a_{\rho}\right)$ immer rational ist. Die Gleichung (40) zeigt sodann, dass auch $\mathbf{R}(z)$ in (39) rational ist.

$\mathrm{Zu}$ diesem Zwecke betrachten wir den Zähler von $\mathbf{R}\left(z ; a_{p}\right)$ :

$$
\begin{gathered}
R\left(z ; a_{\rho}\right)=\mathbf{r}_{0}(z) S\left(z ; a_{\rho}\right)-\mathbf{r}_{1}(z) S\left(z+1 ; a_{\rho}\right) \\
=\mathbf{r}_{0}(z)\left(\frac{A_{y^{\prime}}^{(n)}}{(z-a)^{n^{\prime}}}+\ldots+\frac{A_{1}^{(0)}}{z-a}\right) \\
+\sum_{n=1}^{\infty}\left[\mathbf{r}_{0}(z)\left(\frac{A_{\mu^{\prime}}^{(n)}}{(z-a+n)^{z^{\prime}}}+\ldots+\frac{A_{1}^{(n)}}{z-a+n}\right)-\mathbf{r}_{1}(z)\left(\frac{A_{n^{\prime}}^{(n-1)}}{(z-a+n)^{n^{\prime \prime}}}+\ldots+\frac{A_{1}^{(n-1)}}{z-a+n}\right)\right],
\end{gathered}
$$

wo der Kürze halber

gesetzt sind.

$$
A_{\mu_{\rho}-k i}^{(\rho, n)}=A_{\mu^{\prime}-k}^{(\mu)}, \quad a_{\rho}=a, \quad \mu_{\rho}=\mu^{\prime}
$$

Weil $\mathbf{r}_{0}(z)$ für $z=a$ Null der Ordnung $\mu^{\prime}$ wird, so kann das erste Theil der rechten Seite gleich einer ganzen rationalen Function gesetzt werden, deren Gradzahl höchstens $\mu-\mathrm{I}$ ist, wenn $\mu$ wie in $\S$ I die Gradzahl von $\mathbf{r}_{0}(z)$ bezeichnet. Da $R\left(z ; a_{\rho}\right)$ den Charakter einer ganzen Function hat, so kann auch keines von den Gliedern des zweiten Theiles unendlich werden. Wird also das allgemeine Glied nach ganzen Potenzen von $z-a+n$ entwickelt, so müssen die Coefficienten der nega- 
tiven Potenzen von $z-a+n$ gleich Null werden. Weil dic Gradzahl $\mu$ von $\mathbf{r}_{0}(z)$ nicht kleiner ist als die Gradzahl $\nu$ von $\mathbf{r}_{1}(z)$, so ist offenbar der Exponent der höchsten positiven Potenz von $z-a+n$, die im Gliede überhaupt erscheinen kann, gleich $\mu-\mathrm{I}$. Das allgemeine Glied kann mithin auf die Form

$$
A_{0}+A_{1}(z-a+n)+\ldots+A_{p-1}(z-a+n)^{\mu-1}
$$

gebracht werden. Wird dieser Ausdruck nach Potenzen von $z$ entwickelt, so folgt

$$
R\left(z ; a_{\rho}\right)=\sum_{n=0}^{\infty}\left(B_{0}^{(n)}+B_{1}^{(n)} z+\ldots+B_{\mu-1}^{(n)} z^{n-1}\right)
$$

Da dic Reihe $S\left(z ; a_{\rho}\right)$ gleichmässig convergirt, so ist dies auch mit $R\left(z ; a_{\rho}\right)$ der Fall. Man darf also diese Reihe nach Potenzen von $z$ entwickeln und erhält somit ein Resultat der Form

$$
R\left(z ; a_{\rho}\right)=\alpha_{1}^{(\rho)}+\alpha_{2}^{(\rho)} z+\ldots+\alpha_{\mu}^{(\rho)} z^{\prime-1}
$$

wo $\alpha_{1}^{(\rho)}, \alpha_{2}^{(\rho)}, \ldots, \alpha_{\mu}^{(\rho)}$ von $z$ unabhängige Grössen bezeichnen.

Die in Gleichung (34) vorkommende Function $\mathbf{R}\left(z ; a_{p}\right)$ ist 1nithin immer rational und kann in der Form eines Qvotienten, dessen Nenner gleich demjenigen von $\mathbf{r}(z)$ und dessen Zühler eine ganze rationale Function höchstens $(\mu-I)^{\text {ten }}$ Grades ist, dargestellt werden:

$$
\mathbf{R}\left(z ; a_{p}\right)=\frac{R\left(z ; a_{p}\right)}{\mathbf{r}_{1}(z)}
$$

Verınöge der Gleichung (40) gilt das nämliche von der in Gleichung (39) vorkommenden Function $\mathbf{R}(z)$ :

$$
\begin{gathered}
\mathbf{R}(z)=\frac{h(z)}{\mathbf{r}_{1}(z)} \\
R(z)=\sum_{p=1}^{r} R\left(z ; a_{\rho}\right)=\alpha_{1}+a_{2} z+\ldots+\alpha_{\mu} z^{a-1} .
\end{gathered}
$$

Der Beweis, dass jede Reihe $S\left(z ; a_{p}\right)$ der Form (32), deren Con- 
Hj. Mellin.

stanten $A$ dic Bedingungen (26) erfüllen, unbedingt und gleichmässig convergire, wurde unter der Voraussetzung geführt dass

$$
\lim _{z=\infty}|\mathbf{r}(z)|=|M|>\mathbf{I}
$$

sei. In diesem Falle ist aber nach $\S$ I

$$
\lim _{m=\infty}(z, m)=\infty
$$

woraus sich ergiebt, da offenbar

$$
\lim _{m=\infty} S\left(z+m ; a_{\rho}\right)=0
$$

ist, dass $S\left(z ; a_{\rho}\right)$ nicht nur die Eigenschaft

$$
S\left(z+1 ; a_{p}\right)=\mathbf{r}(z) S\left(z ; a_{r}\right)-\mathbf{R}\left(z ; a_{p}\right)
$$

sondern auch die folgende besitzt:

$$
\lim _{m=\infty} \frac{S\left(z+m ; a_{\rho}\right)}{(z, m)}=0
$$

Hieraus geht nun ferner hervor, dass auch jede in der Form (38) oder (4I) ausdrückbarc Function $S(z)$ den beiden Gleichungen

$$
\begin{gathered}
S(z+1)=\mathbf{r}(z) S(z)-\mathbf{R}(z) \\
\lim _{m=\infty} \frac{S(z+n)}{(z, m)}=0
\end{gathered}
$$

genügt. Es fragt sich nun, ob jede beliebige Function mit diesen beiden Eigenschaften in der Form (38) oder (4I) darstellbar sei. Mit dieser Frage hüngt eine andere zusammen. Wir können zwar unbeschränkt viele Functionen der Form $S(z)$ bilden, von denen jede zwei Gleichungen der oben angegebenen Form befriedigt, wo $\mathbf{R}(z)$ eindeutig bestimmt ist, sobald $S(z)$ gebildet ist. Ist es aber, wenn die rationale Function $\mathbf{R}(z)$ beliebig gegeben ist, auch ungekehrt möglich eine Function $S(z)$ herzustellen, welche diesen beiden Gleichungen genügt? - Indem wir diese Fragen beantworten erhalten wir einen neuen allgemeinen Ausdruck für die in der Form (38) oder (4I) darstellbaren Functionen. 
$\%$.

Zunächst wollen wir den folgenden Satz beweisen:

Jeder beliebigen rationalen Function $\mathbf{R}(z)$ entspricht cine und zwar nur eine einzige Function $S(z)$ mit den beiden Eigenschaften

$$
\left\{\begin{array}{c}
S(z+\mathrm{I})=\mathbf{r}(z) S(z)-\mathbf{R}(z) \\
\lim _{m=\infty} \frac{S(z+m)}{(z, m)}=0 .
\end{array}\right.
$$

Durch wiederholte Anwendung der ersten dieser Gleichungen ergiebt sich

$$
\frac{S(z+m)}{\mathbf{r}(z) \mathbf{r}(z+1) \ldots \mathbf{r}(z+m-1)}
$$

$$
=S(z)-\left(\frac{\mathbf{R}(z)}{\mathbf{r}(z)}+\frac{\mathbf{R}(z+1)}{\mathbf{r}(z) \mathbf{r}(z+1)}+\ldots+\frac{\mathbf{R}(z+m-1)}{\mathbf{r}(z) \mathbf{r}(z+1) \ldots \mathbf{r}(z+m-1)}\right) .
$$

Weil

$$
\frac{S(z+m)}{\mathbf{r}(z) \ldots \mathbf{r}(z+m-\mathrm{I})}=\frac{S(z+m)}{(z, m)} \cdot \frac{(z, m)}{\mathbf{r}(z) \ldots \mathbf{r}(z+m-\mathrm{I})}
$$

ist, und nach § I

$$
\lim _{m=\infty} \frac{(z, m)}{\mathbf{r}(z) \mathbf{r}(z+1) \ldots \mathbf{r}(z+m-1)}=F(z),
$$

so zeigt die zweite der Gleichungen (47), dass die linke Seite von (48) für wachsendes $m$ die Null zur Grenze hat, wenn $z$ von den Unendlichkeitsstellen der Function $F(z)$ verschieden ist. Wenn also eine Function mit den Eigenschaften (47) überhaupt existirt, so ist sie eindeutig bestimmt durch die Gleichung

$$
S(z)=\sum_{n=0}^{\infty} \frac{\mathbf{R}(z+u)}{\mathbf{r}(z) \mathbf{r}(z+\mathbf{1}) \ldots \mathbf{r}(z+n)}
$$

Soll hiermit die Existenz einer Function mit den genannten Eigenschaften 
erwiesen sein, so muss die rechte Seite von (49) eine convergirende Reihe bilden, für welche diese Eigenschaften nachgewiesen werden können.

Da der Qvotient eines Gliedes der Reihe $S(z)$ durch das vorhergehende gleich

$$
\frac{\mathbf{R}(z+n)}{\mathbf{R}(z+n-1)} \cdot \frac{\mathrm{I}}{\mathbf{r}(z+n)}
$$

ist, weil ferner in diesem Ausdrucke

$$
\lim _{n=\infty} \frac{\mathbf{R}(z+n)}{\mathbf{R}(z+n-\mathrm{I})}=\mathrm{I}
$$

und

$$
\lim _{n=\infty}\left|\frac{\mathrm{I}}{\mathbf{r}(z+n)}\right|=\left|\frac{\mathrm{I}}{M}\right|<\mathrm{I}
$$

ist, so ergiebt sich durch ganz gewöhnliche Betrachtungen, dass $S(z)$ eine unbedingt und gleichmässig convergirende Reihe ist. Sie stellt also eine analytische Function dar, die offenbar den Charakter einer rationalen Function hat. Aus der einfachen Bildungsweise der Glieder geht ferner hervor, dass sic die erste der Gleichungen (47) befriedigt, wovon die Gleichung (48) eine Folge ist. Aus dieser und den beiden darauf folgenden Gleichungen findet man, dass sic auch der zweiten von den Gleichungen (47) Genüge leistet.

Im Folgenden betrachten wir nun die Reihe (49) nur unter der Annahme, dass $\mathbf{R}(z)$ eine rationale Function bezeichnet, welche auf dia Form

$$
\mathbf{R}(z)=\frac{R(z)}{\mathbf{r}_{1}(z)}
$$

gebracht werden kann, wo der Zähler $R(z)$ eine ganze rationale Function höchstens $(\mu-1)^{\text {ten }}$ Grades ist:

$$
R(z)=\alpha_{1}+\alpha_{2} z+\ldots+\alpha_{n} z^{\mu-1} .
$$

Indem man den Coefficienten $\alpha_{1}, \alpha_{2}, \ldots, \alpha_{n}$ verschiedene Werthe zuertheilt, bekommt man unendlich viele Functionen der Form (49), ron denen jede einem bestimmten Gleichungssysteme (47) Genüge leistet. Zu diesen gehören alle Functionen, die in der Form (38) oder (4I) dargestellt werden können. Es bleibt noch übrig zu entscheiden, ob auch 
ungekehrt jede Reihe der Form (49) auf die Form (4I) gebracht werden kann.

Um dies entscheiden zu können muss man sich zunächst davon überzeugen, dass man unter den Functionen der Form (4I) immer $\mu$ verschiedene auswählen kann, zwischen denen keine lineare homogene Gleichung mit constanten Coefficienten stattfindet.

Man verfahre z. B. so, dass man aus der Gruppe $\left(a_{1}\right) \mu_{1}$ Reihen $S\left(z ; a_{1}\right)$ nimmt, für welche die Determinante $\Delta_{1}$ von Null verschieden ist, aus der Gruppe $\left(a_{2}\right) \mu_{2}$ Reihen $S\left(z ; a_{2}\right)$ für welche die Determinante $\Delta_{2}$ von Null verschieden ist, u. s. w., und schliesslich aus der Gruppe $\left(a_{r}\right)^{\prime} \mu_{r}$ Reihen $S\left(z ; a_{r}\right)$ für welche die Determinante $\Delta_{r}$ von Null verschieden ist. Man bekommt so im Ganzen $\mu_{1}+\mu_{2}+\ldots+\mu_{r}=\mu$ Reihen, die wir in einer beliebigen Reihenfolge schreiben und mit

$$
S_{1}(z), \quad S_{2}(z), \quad . \quad \cdot \quad, S_{n}(z)
$$

bezeichnen wollen. Zwischen dicsen Reihen kann nun keine lineare homogene Gleichung mit constanten Coefficienten stattfinden. Denn würde eine solche bestehen, so müsste auch, da zwei zu verschiedenen Gruppen gehörige Reihen keine gemeinschaftliche Unendlichkeitsstelle haben, eine lineare homogene Gleichung mit constanten Coefficienten zwischen den zur Gruppe $\left(a_{\rho}\right),(\rho=1,2, \ldots, r)$, gehörigen Reihen stattfinden, was unmöglich ist, da die Determinante $\Delta_{\rho}$ von Null verschieden ist.

Es seien nun

$$
\begin{gathered}
S_{\lambda}(z+1)=\mathbf{r}(z) S_{\lambda}(z)-\mathbf{R}_{\lambda}(z) \\
\lambda=\mathrm{I}, 2, \ldots, r
\end{gathered}
$$

die $\mu$ Gleichungen, denen beziehungsweise die Reihen (50) genügen; es werde

$$
\begin{gathered}
\mathbf{R}_{\lambda}(z)=\frac{\boldsymbol{R}_{\lambda}(z)}{\mathbf{r}_{1}(z)}, \\
R_{\lambda,}(z)=\alpha_{\lambda, 1}+\alpha_{\lambda, 2} z+\ldots+\alpha_{\lambda, \mu} z^{\prime \prime-1}
\end{gathered}
$$

gesetzt, und die Determinante

$$
\partial=\left|\begin{array}{cccc}
a_{11} & a_{12} & \ldots & a_{1, n} \\
\alpha_{21} & a_{22} & \ldots & \alpha_{2 n} \\
\cdots & \ldots & \ldots & . \\
\alpha_{\mu 1} & \alpha_{\mu 2} & \ldots & a_{\mu \mu}
\end{array}\right|
$$


Hj. Mellin.

gebildet, so gilt der folgende Satz: Wenn die Functionen (50) so gewählt sind, dass zwischen ihnen keine homogene lineare Gleichung mit constanten Coefficienten stattfindet, so kann die Determinante o nicht gleich Null sein.

Denn bildet man die Function

$$
f(z)=p_{1} S_{1}(z)+p_{2} S_{2}(z)+\ldots+p_{\mu} S_{\mu}(z)
$$

so besitzt sie offenbar die Eigenschaft

$$
f(z+\mathbf{1})=\mathbf{r}(z) f(z)-\overline{\mathbf{R}}(z),
$$

wo

$$
\overline{\mathbf{R}}(z)=\frac{\bar{R}(z)}{\mathbf{r}_{1}(z)}
$$

und

$$
\bar{R}(z)=p_{1} R_{1}(z)+p_{2} R_{2}(z)+\ldots+p_{p} R_{n}(z)
$$

ist. Wird $\bar{R}(z)$ nach Potenzen von $z$ entwickelt, so folgt ein Resultat der Form

$$
\bar{R}(z)=\bar{\alpha}_{1}+\bar{\alpha}_{2} z+\ldots+\bar{\alpha}_{n} z^{z-1} \text {. }
$$

Wäre nun $\partial=0$ so könnte dem homogenen linearen Gleichungssystem

$$
\begin{aligned}
& \bar{\alpha}_{1}=\alpha_{11} p_{1}+\alpha_{21} p_{2}+\ldots+\alpha_{\mu 1} p_{\mu}=0 \\
& \bar{\alpha}_{2}=\alpha_{12} p_{1}+\alpha_{22} p_{2}+\ldots+\alpha_{\mu 2} p_{\mu}=0 \\
& \cdot \cdot \cdot \cdot \cdot \cdot \cdot \cdot \cdot \cdot \cdot \cdot \cdot \cdot \alpha_{\mu \mu} p_{\mu}=0
\end{aligned}
$$

ein Werthsystem

$$
p_{1}, p_{2}, \ldots, p_{n}
$$

genügen, wo die sämmtlichen $p$ nicht gleich Null sind. Alsdann würde aber sein

$$
f(z+\mathbf{1})=\mathbf{r}(z) f(z)
$$

und mithin

$$
\frac{f(z+m)}{\mathbf{r}(z) \mathbf{r}(z+1) \ldots \mathbf{r}(z+m-1)}=f(z),
$$


wie gross auch die positive ganze Zahl $m$ sein mag. Es müsste also wegen der Relationen

$$
\lim _{m=\infty} f(z+m)=0, \quad \lim _{z=\infty}|\mathbf{r}(z)|>\mathrm{I}
$$

$f(z)$ identisch verschwinden, mit andern Worten die Gleichung

$$
f(z)=p_{1} S_{1}(z)+p_{2} S_{2}(z)+\ldots+p_{n} S_{n}(z)=0
$$

für alle Werthe der Veränderlichen $z$ stattfinden. Dies stimmt aber nicht überein mit unserer Annahme, dass zwischen den Functionen $S$ keine lineare homogene Gleichung mit constanten Coefficienten stattfindet. Es muss also $\partial$ von Null verschieden sein, wenn die Functionen $S$ linear unabhängig sind.

Wir wollen jetzt nachweisen, dass jede Reihe der Form (49), wo

$$
\begin{gathered}
\mathbf{R}(z)=\frac{R(z)}{\mathbf{r}_{1}(z)}, \\
R(z)=\alpha_{1}+\alpha_{2} z+\ldots+\alpha_{\mu} z^{\mu-1}
\end{gathered}
$$

ist, auf die Form. (4I) gebracht werden kann. Es seien wie früher

$$
S_{1}(z), S_{2}(z), \ldots, S_{\mu 2}(z)
$$

/ Reihen (50), wwischen denen keine lineare homogene Gleichung mit constanten Coefficienten stattfindet, und man bilde den Ausdruck

$$
f(z)=p_{1} S_{1}(z)+p_{2} S_{2}(z)+\ldots+p_{p} S_{\mu}(z) .
$$

Da die Determinante $\partial$ des linearen Gleichungssystems

$$
\bar{\alpha}_{1}=\alpha_{1}, \bar{\alpha}_{2}=\alpha_{2}, \ldots, \bar{\alpha}_{\mu}=\alpha_{n}
$$

nach dem vorstehenden Satze von Null verschieden ist, so giebt es ein und zwar nur ein Werthsystem

$$
p_{1}, p_{2}, \ldots, p_{\mu},
$$

welches den Gleichungen genügt, für welches mithin

$$
\bar{R}(z)=R(z)
$$


Hj. Mollin.

ist. Für dieses Werthsystem sind nun auch die Brüche $\overline{\mathbf{R}}(z)$ und $\mathbf{R}(z)$ gleich, da sie denselben Nenner haben, und es ist also bewiesen, dass die Functionen $f(z)$ die erste der Gleichungen (47) befriedigt, denen $S(z)$ Genüge leistet. Sie befriedigt aber offenbar auch die zweite. Nach dem im Anfange dieses $\$$ bewiesenen Satze ist also

$$
f(z)=S(z) \text {. }
$$

Hiermit ist nun folgender Satz bewiesen:

Sind

$$
S_{1}(z), S_{2}(z), \ldots, S_{\mu}(z)
$$

$\mu$ Functionen der Form (37) oder (4I), welche so gewählt sind, dass zwischen ihnen keine lineare homogene Gleichung mit constanten Coefficienten stattfindet, so kann jede Function $S(z)$ mit den beiden Eigenschaften (47), wo

$$
\mathbf{R}(z)=\frac{\alpha_{1}+\alpha_{2} z+\ldots+\alpha_{p} z^{\mu-1}}{\mathbf{r}_{1}(z)}
$$

ist, immer und zwar nur auf eine Weise in der Form

$$
S(z)=p_{1} S_{1}(z)+p_{2} S_{2}(z)+\ldots+p_{i h} S_{u}(z)
$$

wo $p_{1}, p_{2}, \ldots, p_{\mu}$ Constanten bezeichnen, dargestellt werden.

Im Vorstehenden ist bewiesen worden, dass die Determinante $\partial$ einen von Null verschiedenen Werth hat, wenn die Functionen $S_{1}(z), S_{2}(z), \ldots, S_{\mu}(z)$ so gewählt sind, dass zwischen ihnen keine lineare homogene Gleichung mit constanten Coefficienten stattfindet. Aus den dabei angewandten Betrachtungen ergiebt sich aber, dass eine solche Gleichung besteht, wenn $\grave{o}=0$ ist. Eine nothwendige und hinreichende Bedingung dafiur, dass die Functionen $S$ linear unabhängig seien, ist mithin, dass die Determinante ò einen von Null verschiedenen Werth habe.

8.

Es sei $\mathbf{R}(z)$ eine rationale Function der Form

$$
\mathbf{R}(z)=\frac{\alpha_{1}+\alpha_{2} z+\ldots+\alpha_{\mu} z^{\mu-1}}{\mathbf{r}_{1}(z)},
$$


wo $\alpha_{1}, \alpha_{2}, \ldots, \alpha_{\mu}$ als unbestimmte Grössen betrachtet werden, und es mag ein particulares Integral des Systems der Functionalgleichungen

$$
\left\{\begin{array}{c}
S(z+\mathbf{1})=\mathbf{r}(z) S(z)-\mathbf{R}(z) \\
\lim _{m=\infty} \frac{S(z+m)}{(z, m)}=0
\end{array}\right.
$$

jede Function heissen, die einem der unendlich vielen Gleichungssystemen genügt, worin das System (52) übergehen kann, wenn $\mathbf{R}(z)$ als eine specielle rationale Function der angegebenen Form aufgefasst wird. Alsdann hat man den folgenden Satz, der mit einem fundamentalen Satze aus der Theorie der linearen Differentialgleichungen vollständig übereinstimmt.

Ist

$$
S_{1}(z), S_{2}(z), \ldots, S_{n}(z)
$$

ein solches System particulärer Integrale des obigen Systems von Functionalgleichungen, dass seine Determinante o einen von Null verschiedenen Werth hat, so kann jedes andere Integral $S(z)$ desselben Systems als lineare homogene Function mit constanten Coefficienten von $S_{1}(z), S_{2}(\ddot{z}), \ldots, S_{\mu}(z)$ ausgedrückt werden:

$$
S(z)=p_{1} S_{1}(z)+p_{2} S_{2}(z)+\ldots+p_{\mu} S_{\mu}(z)
$$

Da der Ausdruck (54) durch eine geeignete Specialisirung der Constanten $p_{1}, p_{2}, \ldots, p_{i}$ gleich jeder beliebigen Functionen gemacht werden kann, die einem speciellen Gleichungssysteme der Form (52) genügt, so nennen wir diesen Ausdruck das allgemeine Integral des Systems der Functionalgleichungen (52). Wird ferner mit einem Fundamentalsystem particulärer Integrale jedes System (53) bezeichnet, von dessen Elementen jedes Integral von (52) als lincare homogene Function ausgedrückt werden kann, so gilt noch der folgende Satz:

Die particulären Integrale

$$
S_{1}(z), S_{2}(z), \ldots, S_{n}(z)
$$

constituiren ein Fundamentalsystem einzig und allein in dem Falle wo keine 
lineare homogene Gleichung mit constanten Coefficienten zwischen ihnen stattfinden kann.

Schliesslich hat man den folgenden Satz: Zwischen je $\mu+\mathrm{x}$ particulïren Integralen des Systems (52) besteht eine lineare homogene Gleichung mit constanten Coefficienten.

9.

Wir betrachten jetzt die Gleichung

$$
F(z)=P(z)+Q(z),
$$

wo $P(z)$ die zu $F(z)$ gehörige Partialbruchreihe und $Q(z)$ eine beständig convergirende Potenzreihe bezeichnet. Da die Function $P(z)$ nach den vorigen Untersuchungen ein particulüres Integral des Gleichungssystems (52) ist, so hat man

$$
P(z+1)=\mathbf{r}(z) P(z)-\mathbf{R}^{*}(z),
$$

wo $\mathbf{R}^{*}(z)$ eine gewisse rationale Function der Form

$$
\mathbf{R}^{*}(z)=\frac{\alpha_{1}+\alpha_{2} z+\ldots+\alpha_{\mu} z^{n-1}}{\mathbf{r}_{1}(z)}
$$

bezeichnet. Stellt man die Gleichung $F(z+1)=\mathbf{r}(z) F(z)$ mit $(55)$ und (56) zusammen, so findet man, dass die beständig convergirende Potenzreihe

der Gleichung

$$
Q(z)=c_{0}+c_{1} z+\ldots+c_{n} z^{n}+\ldots
$$

$$
Q(z+1)=\mathbf{r}(z) Q(z)+\mathbf{R}^{*}(z)
$$

genügt.

Die Potenzreihe $Q(z)$ kann dem System der Functionalgleichungen (52) nicht genügen. Das genannte System ist ein Specialfall des folgenden

$$
\left\{\begin{array}{c}
S(z+\mathrm{I})=\mathbf{r}(z) S(z)-\mathbf{R}(z) \\
\lim _{m=\infty} \frac{S(z+m)}{(z, m)}=K,
\end{array}\right.
$$


Zur Theorie der Gammafunction.

wo $K$ eine unbestimmte Constante und $\mathbf{R}(z)$ eine allgemeine rationale Function der in vorigem $\S$ angegebenen Form bezeichnet. Dass $Q(z)$ ein particuläres Integral des Systems (58) ist, geht aus dem folgenden Satze hervor:

Jeder Combination einer bestimmten Constante $K$ und einer speciellen rationalen Function $\mathbf{R}(z)$ der oft genannten Form entspricht eine und awar nur eine einzige Function $S(z)$ mit den beiden Eigenschaften (58). Es ist ferner immer und nur auf eine Weise möglich die Constanten $p_{1}, p_{2}, \ldots, p_{\mu}, q$ so zu bestimmen, dass

$$
S(z)=p_{1} S_{1}(z)+p_{2} S_{2}(z)+\ldots+p_{n} S_{n}(z)+q Q(z)
$$

wird, wo $S_{1}(z), S_{2}(z), \ldots, S_{n}(z)$ ein Fundamentalsystem particulürer Integrale des Systems der Functionalgleichungen (52) constituiren.

Durch wiederholte Anwendung der ersten der Gleichungen (58) erhält man

$$
\begin{gathered}
\frac{S(z+m)}{\mathbf{r}(z) \mathbf{r}(z+\mathrm{I}) \ldots \mathbf{r}(z+m-\mathbf{I})} \\
=S(z)-\left(\frac{\mathbf{R}(z)}{\mathbf{r}(z)}+\frac{\mathbf{R}(z+\mathrm{I})}{\mathbf{r}(z) \mathbf{r}(z+\mathrm{I})}+\ldots+\frac{\mathbf{R}(z+m-1)}{\mathbf{r}(z) \mathbf{r}(z+\mathrm{I}) \ldots \mathbf{r}(z+m-\mathrm{I})}\right) .
\end{gathered}
$$

Wendet man die zweite Gleichung an, so folgt

$$
\begin{gathered}
\lim _{m=\infty} \frac{S(z+m)}{\mathbf{r}(z) \mathbf{r}(z+\mathrm{I}) \ldots \mathbf{r}(z+m-\mathrm{I})} \\
=\lim _{m=\infty} \frac{S(z+m)}{(z, m)} \cdot \frac{(z, m)}{\mathbf{r}(z) \mathbf{r}(z+\mathrm{I}) \ldots \mathbf{r}(z+m-\mathrm{I})}=K \cdot F(z),
\end{gathered}
$$

wenn auch die Gleichung (9) in $\S$ I in Betracht gezogen wird, und es muss mithin

$$
S(z)=K F(z)+\sum_{n=0}^{\infty} \frac{\mathbf{R}(z+n)}{\mathbf{r}(z) \mathbf{r}(z+1) \ldots \mathbf{r}(z+n)}
$$

sein. Wenn also eine Function mit den beiden Eigenschaften (58) existirt, so ist sie in dieser Form darstellbar. Da die rechte Seite der letzten Gleichung eine Function wirklich darstellt, welche die beiden angenommenen Eigenschaften der linken Seite offenbar besitzt, so ist hiermit der 
Hj. Mellin.

erste Theil des obigen Satzes bewiesen. Setzt man ferner auf der rechten Seite

$$
F(z)=P(z)+Q(z)
$$

so folgt auch die Richtigkeit des zweiten Theiles, wenn man bemerkt, dass es immer und zwar nur auf eine Weise möglich ist, die Constanten $p_{1}, p_{2}, \ldots, p_{k}$ so zu bestimmen, dass

$$
K P(z)+\sum_{n=0}^{\infty} \frac{\mathbf{R}(z+n)}{\mathbf{r}(z) \mathbf{r}(z+\mathrm{I}) \ldots \mathbf{r}(z+n)}=p_{1} S_{1}(z)+p_{2} S_{2}(z)+\ldots+p_{\mu} S_{\mu}(z)
$$

wird.

Da also der Ausdruck

$$
S(z)=p_{1} S_{1}(z)+p_{2} S_{2}(z)+\ldots+p_{n} S_{n}(z)+K Q(z)
$$

durch eine geeignete Specialisirung der Constanten $p_{1}, p_{2}, \ldots, p_{n}, K$ gleich jeder beliebigen Function gemacht werden kann, die einem speciellen Gleichungssysteme der Form (58) genügt, so ist dieser Ausdruck das allgemeine Integral des Systems der Functionalgleichungen (58).

Nimmt man an, es sei $K=\mathbf{1}$, und bestimmt die Constanten $p$ so, dass

$$
p_{1} S_{1}(z)+p_{2} S_{2}(z)+\ldots+p_{n} s_{n}(z)=P(z)
$$

ist, so wird

$$
S(z)=F(z)
$$

Nimmt man an, es sei $K=0$, und bestimmt die Constanten $p$ wie früher, so wird

$$
S(z)=P(z)
$$

Nimmt man an, es sei $K=\mathrm{I}$, und setzt $p_{1}=p_{2}=\ldots=p_{\mu}=\mathrm{o}$, so wird

$$
S(z)=Q(z)
$$

Es sind also $F(\ddot{*}), P(\xi), Q(\varepsilon)$ particuläre Integrale des allgemeinen Gleichungasystems (58).

Aus dem soeben bewiesenen Satze geht nun auch die Richtigkeit des folgenden hervor: 
Die Function $F(z)$, welche die charakteristischen Eigenschaften

$$
F(z+\mathrm{I})=\mathbf{r}(z) F(z), \quad \lim _{m=\infty} \frac{F(z+m)}{(z, m)}=\mathrm{I}
$$

besitzt, kann als eine Summe zweier anderen Functionen $P(z)$ und $Q(z)$ dargestellt werden, von denen $P(z)$ eine Partialbruchreihe der Form (38) mit den charakteristischen Eigenschaften

$$
P(z+\mathrm{I})=\mathbf{r}(z) P(z)-\mathbf{R}^{*}(z), \quad \lim _{m=\infty} \frac{P(z+m)}{(z, m)}=0
$$

und $Q(z)$ eine beständig convergirende Potenzreihe mit den charakteristischen Eigenschaften

bezeichnet.

$$
Q(z+\mathbf{I})=\mathbf{r}(z) Q(z)+\mathbf{R}^{*}(z), \quad \lim _{m=\infty} \frac{Q(z+m)}{(z, m)}=\mathbf{I}
$$

Unter den particulären Integralen des Systems (58) sind $F(z), P(z)$, $Q(z)$ besonders bemerkenswerth und zwar aus folgenden Gründen.

$F(z)$ ist, abgesehen von einem constanten Factor, die einzige Function, welche dem Gleichungssystem (58) genügt, wenn $\mathbf{R}(z)=$ o ist.

Das allgemeine Integral geht nur dann in $F(z)$ über, wenn man $K=\mathrm{I}$ und

setzt.

$$
p_{1} S_{1}(z)+p_{2} S_{2}(z)+\ldots+p_{\mu} S_{n}(z)=P(z)
$$

$Q(z)$ ist, abgesehen von einem constanten Factor, die einzige Function mit dem Charakter einer ganzen Function, welche dem Gleichungssystem (58) genügt, und dann muss $\mathbf{R}(z)=-\mathbf{R}^{*}(z)$ sein. Deswegen ist auch die rationale Function $\mathbf{R}^{*}(z)$ besonders bemerkenswerth.

10.

Nach den vorigen Untersuchungen entspricht jeder Function $S(z)$ der Form (38) eine rationale Function der Form

$$
\mathbf{R}(z)=\frac{\alpha_{1}+\alpha_{2} z+\ldots+\alpha_{\mu} z^{\mu-1}}{\mathbf{r}_{1}(z)}
$$

Acto mathematica. 8. Imprimé le 6 Mars 1886 , 
für welche die Gleichung

$$
S(z+1)=\mathbf{r}(z) S(z)-\mathbf{R}(z)
$$

stattfindet. Umgekehrt entspricht auch jeder rationalen Function der genannten Form eine Function $S(z)$, welche dieser Gleichung genügt. Es ist noch übrig zu zeigen, wie die eine dieser Functionen bestimmt werden soll, wenn die andere gegeben ist.

Es mag zunächst angenommen werden, dass $S(z)$ gegeben ist und dass $\mathbf{R}(z)$ bestimmt werden soll. Es sei $a$ irgend ein Werth, für den weder $S(z)$ noch $S(z+\mathrm{I})$ unendlich wird. Werden $\mathbf{r}_{0}(z), \mathbf{r}_{\mathbf{1}}(z), S(z)$, $S(z+1)$ nach positiven ganzzahligen Potenzen von $z-a$ entwickelt und der Zühler von $\mathbf{R}(z)$

$$
R(z)=\mathbf{r}_{0}(z) S(z)-\mathbf{r}_{1}(z) S(z+\mathbf{1})
$$

nach wachsenden Potenzen von $z-a$ geordnet, so werden die Coefficienten derjenigen Potenzen, deren Exponenten grösser als $\mu-\mathrm{I}$ sind, gleich Null. Setzt man

$$
R(z)=A_{1}+A_{2}(z-a)+\ldots+A_{m}(z-a)^{k-1},
$$

so ist offenbar

$$
\begin{gathered}
A_{\lambda+1}=\mathbf{r}_{0}(a) \frac{S^{(\lambda)}(a)}{\mid \underline{\lambda}}+\mathbf{r}_{0}^{\prime}(a) \frac{S^{(\lambda-1)}(a)}{\mid \underline{\lambda-\mathrm{I}}}+\ldots+\frac{\mathbf{r}_{0}^{(\lambda)}(a)}{\mid \underline{\underline{\lambda}}} S(a) \\
-\left(\mathbf{r}_{1}(a) \frac{S^{(\lambda)}(a+\mathrm{I})}{\mid \underline{\lambda}}+\mathbf{r}_{1}^{\prime}(a) \frac{S^{(\lambda-1)}(a+\mathrm{I})}{\mid \underline{\lambda-\mathrm{I}}}+\ldots+\frac{\mathbf{r}_{1}^{(\lambda)}(a)}{\mid \underline{\lambda}} S(a+\mathrm{I})\right) . \\
(\lambda=\mathrm{o}, \mathrm{I}, \ldots, \mu-\mathrm{I}) .
\end{gathered}
$$

Hat man in dieser Weise für die Elemente

$$
S_{1}(z), S_{2}(z), \ldots, S_{\mu}(z)
$$

eines Fundamentalsystems von (47) die entsprechenden Functionen

$$
\mathbf{R}_{1}(z), \mathbf{R}_{2}(z), \ldots, \mathbf{R}_{\mu}(z)
$$

bestimmt, so ist diejenige rationale Function $\mathbf{R}(z)$, die einem anderen 
beliebig gegebenen particulïren Integrale $S(z)$ entspricht, durch die Gleichung

$$
\mathbf{R}(z)=p_{1} S_{1}(z)+p_{2} S_{2}(z)+\ldots+p_{p} S_{\mu}(z)
$$

gegeben, wenn $p_{1}, p_{2}, \ldots, p_{\mu}$ dasjenige Werthsystem bezeichnet, für welches die Gleichung

$$
S(z)=p_{1} S_{1}(z)+p_{2} S_{2}(z)+\ldots+p_{\mu} S_{\mu}(z)
$$

stattfindet. Die Constanten $p$ ergeben sich durch Auflösung eines Systems von $\mu$ linearen Gleichungen.

Ist $\mathbf{R}(z)$ gegeben, so ist die entsprechende Function $S(z)$ durch die Gleichung

$$
S(z)=\sum_{n=0}^{\infty} \frac{\mathbf{R}(z+n)}{\mathbf{r}(z) \mathbf{r}(z+\mathrm{I}) \ldots \mathbf{r}(z+n)}
$$

unmittelbar bestimmt. Hier tritt die Function $S(z)$ jedoch nicht in der Form einer Partialbruchreihe auf. Hat man aber für ein Fundamentalsystem, dessen Elemente $S_{1}(z), S_{2}(z), \ldots, S_{\mu}(z)$ in dieser Form gegeben sind, die bezüglichen zu den Elementen gehörigen rationalen Functionen $\mathbf{R}_{1}(z), \mathbf{R}_{2}(z), \ldots, \mathbf{R}_{\mu}(z)$ bestimmt, so kann $S(z)$ durch Aufösung eines Systems von $\mu$ linearen Gleichungen auf die Form einer Partialbruchreihe gebracht werden. Es ist nämlich

$$
S(z)=p_{1} S_{1}(z)+p_{2} S_{2}(z)+\ldots+p_{\mu} S_{k}(z),
$$

wenn $p_{1}, p_{2}, \ldots, p_{\mu}$ dasjenige Werthsystem bezeichnet für welches

$$
\mathbf{R}(z)=p_{1} \mathbf{R}_{1}(z)+p_{2} \mathbf{R}_{2}(z)+\ldots+p_{\mu} \mathbf{R}_{\mu}(z) .
$$

11.

Nach dem, was im vorigen $\S$ gezeigt worden ist, braucht es keiner näheren Erörterung, wenn es sich um die Lösung der soeben betrachteten Probleme für das allgemeinere System der Functionalgleichungen (58) handelt. Es sei nur bemerkt, dass alsdann $P(z)$ und die entsprechende rationale Function $\mathbf{R}^{*}(z)$ bestimmt sein müssen. 
Hj. Mellin.

Die Function $P(z)$ ist eine Summe von $r$ Reihen:

$$
P(z)=P\left(z ; a_{1}\right)+P\left(z ; a_{2}\right)+\ldots+P\left(z ; a_{r}\right) .
$$

$\mathrm{Zu}$ jeder dieser $r$ Reihen gehört ein System von Bedingungsgleichungen, denen die Constanten $A$ genügen. Durch diese Gleichungen werden die Constanten des ersten Gliedes nicht bestimmt. Diese sollen jetzt ermittelt werden.

Bezeichnet man die logarithmischen Ableitungen von $F(z)$ und $\Gamma(z)$ beziehungsweise mit $\Psi(z)$ und $\psi(z)$, so ist offenbar

$$
\begin{aligned}
\psi(z)=\alpha & +\mu_{1} \phi\left(z-a_{1}\right)+\mu_{2} \psi\left(z-a_{2}\right)+\ldots+\mu_{r} \phi\left(z-a_{r}\right) \\
& -\nu_{1} \phi\left(z-b_{1}\right)-\nu_{2} \psi\left(z-b_{2}\right)-\ldots-\nu_{s} \phi\left(z-b_{s}\right) .
\end{aligned}
$$

Die Function $\phi(z)$ kann bekanntlich in der Form

$$
\phi(z)=-C-\frac{\mathrm{I}}{z}+\left(\mathrm{I}-\frac{\mathrm{I}}{z+\mathrm{I}}\right)+\left(\frac{\mathrm{I}}{2}-\frac{\mathrm{I}}{z+2}\right)+\ldots
$$

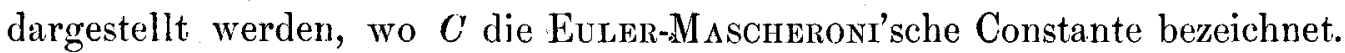
Es sei nun $P\left(z ; a_{\rho}\right)$ eine der Reihen, von denen $P(z)$ eine Summe ist, und es mag der Kürze halber

$$
\hat{A}_{r_{\rho}-k}^{(\rho, 0)}=A_{\mu_{\rho}-k}
$$

gesetzt werden. Durch theilweise Integration erhalten wir nun zunächst aus

$$
A_{n_{p}-k}=\frac{\mathrm{I}}{2 \pi} \int_{0}^{2 \pi} F\left(a_{\rho}+r e^{i t}\right)\left(r e^{i t}\right)^{\mu_{\rho}-k} d t
$$

die Gleichung

$$
A_{\mu_{\rho}-k}=-\frac{\mathrm{I}}{2\left(\mu_{\rho}-k\right) \pi} \int_{0}^{2 \pi} F\left(a_{\rho}+r e^{i t}\right) \Psi\left(a_{\rho}+r e^{i t}\right)\left(r e^{i t}\right)^{\mu_{\rho}-k+1} d t .
$$

Setzt man in Gleichung (6I) $x=a_{p}+r e^{i t}$, so erscheint auf der rechten Seite das Glied $\mu_{p} \psi\left(r e^{i t}\right)$. Setzt man

$$
\mu_{\rho} \psi\left(r e^{i t}\right)=-\frac{\mu_{\rho}}{r e^{i t}}+\mu_{\rho} \psi\left(\mathrm{I}+r e^{i t}\right)
$$


und entwickelt die rechte Seite von (6I) nach ganzen Potenzen von $r e^{i t}$, so bekommt man eine Reihe der Form

$$
\Psi\left(a_{\rho}+r e^{i t}\right)=-\frac{\mu_{\rho}}{r e^{i t}}+\alpha+C_{0}+C_{1} r e^{i t}+\ldots+\frac{C_{\lambda}}{\mid \underline{\lambda}}\left(r e^{i t}\right)^{\lambda}+\ldots
$$

wo

$$
C_{\lambda}=\sum_{\sigma=1}^{r}(\rho) \mu_{\rho} \psi^{(\lambda)}\left(a_{\rho}-a_{\sigma}\right)-\sum_{\sigma=1}^{s} \nu_{\rho} \psi^{(\lambda)}\left(a_{\rho}-b_{\sigma}\right)
$$

ist. Es wird durch $\sum^{(\rho)}$ angedeutet, dass dasjenige Glied, wo $\sigma=\rho$ ist, durch $\mu_{\rho} \psi^{(2)}(\mathrm{I})$ zu ersetzen ist. Setzt man in (62) statt $\Psi\left(a_{\rho}+r e^{i t}\right)$ die Reihenentwickelung $\left(6_{3}\right)$ ein und führt die Integration gliedweise aus, indem man beachtet, dass

$$
\int_{0}^{2 \pi} F\left(a_{\rho}+r e^{i t}\right)\left(r e^{i t}\right)^{h} d t=0
$$

für $h=\mu_{\rho}+\mathrm{I}, \mu_{\rho}+2, \ldots$, so ergiebt sich

$$
k A_{\mu_{\rho}-k}=\left(\alpha+C_{0}\right) A_{\mu_{g}-k+1}+C_{1} A_{\mu_{\rho}-k+2}+\ldots+\frac{C_{k-1}}{\underline{k-1}} A_{\mu_{p}} .
$$

Für $k=\mathbf{I}, 2, \ldots, \mu_{\rho}-\mathrm{I}$ bekommt man die Recursionsformeln

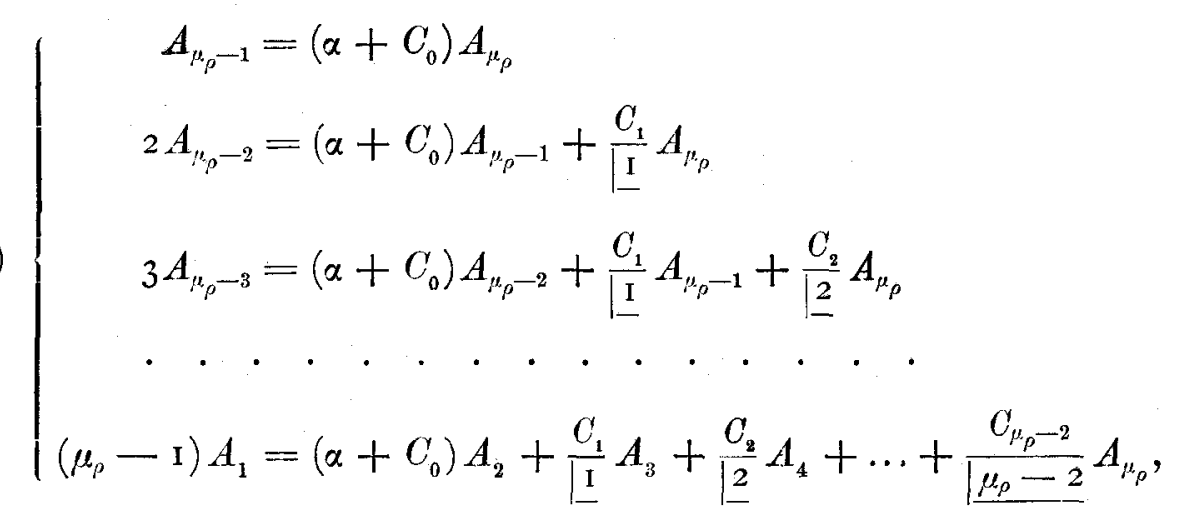

wo

$$
\begin{gathered}
A_{\mu_{\rho}}=\lim _{z=a_{\rho}}\left(z-a_{\rho}\right)^{\mu_{\rho}} F(z) \\
=e^{\alpha a_{\rho}} \frac{\Gamma^{\mu_{1}}\left(a_{\rho}-a_{1}\right) \ldots \Gamma^{\nu_{\rho}-1}\left(a_{\rho}-a_{\rho-1}\right) \Gamma^{u_{\rho}+1}\left(a_{\rho}-a_{\rho+1}\right) \cdots \Gamma^{u_{r}}\left(a_{\rho}-a_{r}\right)}{\Gamma^{\nu_{1}}\left(a_{\rho}-b_{1}\right) \Gamma^{\nu_{2}}\left(a_{\rho}-b_{2}\right) \ldots \Gamma^{\nu r}\left(a_{\rho}-b_{s}\right)} .
\end{gathered}
$$


$1 \%$.

Die in den $\$ \S 5-$ I I angestellten Untersuchungen sind unter der Voraussetzung geführt worden, dass

$$
\lim _{z=\infty}|\mathbf{r}(z)|>\mathrm{I}
$$

ist. Den interessanten Fall, wo

$$
\lim _{z=\infty}|\mathbf{r}(z)|=\mathbf{I}
$$

ist, will ich bei einer anderen Gelegenheit behandeln.

Es findet cine enge Beziehung statt zwischen den Integralen gewisser linearen Differentialgleichungen und solchen Transcendenten, von denen im Vorigen die Rede gewesen ist. Die Beschaffenheit dieser Beziehung soll hier kurz angegeben werden. Die zur Function

$$
F(z)=\Gamma^{\mu}(z)
$$

gehörigen Reihen $S(z)$ bilden eine Gruppe und haben alle die Form

$$
S(z)=\sum_{n=0}^{\infty}\left(\frac{A_{\mu}^{(n)}}{(z+n)^{\mu}}+\frac{A_{\mu-1}^{(n)}}{(z+n)^{\mu-1}}+\ldots+\frac{A_{1}^{(n)}}{z+n}\right) .
$$

Jede derselben befriedigt eine Gleichung

$$
S(z+\mathrm{I})=z^{\mu} S(z)-\mathbf{R}(z),
$$

wo $\mathbf{R}(z)$ eine ganze rationale Function höchstens $(\boldsymbol{\mu}-\mathrm{I})^{\text {ten }}$ Grades bezeichnet. Die Constanten $A$ genügen den Bedingungen

$A_{p}^{(n)}=\left(\frac{-\mathrm{I}}{n}\right)^{\mu} A_{\mu}^{(n-1)}$

$$
\begin{aligned}
& A_{\mu-k}^{(n)}=\left(\begin{array}{c}
-\mu \\
k
\end{array}\right)\left(\frac{-\mathrm{I}}{n}\right)^{\mu+k} A_{\mu-1}^{(n-1)}+\left(\begin{array}{c}
-\mu \\
k-1
\end{array}\right)\left(\frac{-1}{n}\right)^{\mu+k-1} A_{\mu-1}^{(n-1)}+\ldots+\left(\frac{-\mathrm{I}}{n}\right)^{\mu} A_{k-k}^{(n-1)} \\
& A_{1}^{(n)}=\left(\begin{array}{c}
-\mu \\
\mu-\mathrm{I}
\end{array}\right)\left(\frac{-\mathrm{I}}{n}\right)^{2 \mu-1} A_{\mu}^{(n-1)}+\left(\begin{array}{c}
-\mu \\
\mu-2
\end{array}\right)\left(\frac{-\mathrm{I}}{n}\right)^{2 \mu-2} A_{\mu-1}^{(n-1)}+\ldots+\left(\frac{-\mathrm{I}}{n}\right)^{\mu} A_{1}^{(n-1)} \text {. }
\end{aligned}
$$


Setzt man

$$
A_{\mu}^{(0)}=\mathrm{o}, \quad A_{p-1}^{(0)}=\mathrm{o}, \ldots . \quad A_{1}^{(0)}=\mathrm{I},
$$

so bekommt man eine Reihe der Form

$$
S(z)=\sum_{n=0}^{\infty} \frac{(-1)^{\mu n}}{(\mid \underline{n})^{\mu}} \cdot \frac{\mathrm{I}}{z+n} .
$$

Setzt man ferner

$$
y=\sum_{n=0}^{\infty} \frac{(-\mathrm{I})^{u n}}{(\mid \underline{n})^{n}} x^{n}
$$

so kann $S(z)$, wenn der reelle Theil von $z$ grösser als Null ist, folgendermaassen in der Form eines bestimmten Integrals ausgedrückt werden:

$$
S(z)=\int_{0}^{1} y x^{z-1} d x
$$

Die beständig convergirende Potenzreihe $y$ genügt, wie sich leicht ergiebt, der linearen homogenen Differentialgleichungen $\mu^{\text {ter }}$ Ord́nung

$$
D_{x} x D_{x} \ldots x D_{x} y=(-1)^{\mu} y .
$$

Es gilt nun folgender Satz:

Jedem Fundamentalsystem particulärer Integrale

$$
S_{1}(z), S_{2}(z), \ldots, S_{p}(z)
$$

des Systems der Functionalgleichungen

$$
\left\{\begin{array}{c}
S(z+\mathrm{I})=z^{\prime \prime} S(z)-\mathbf{R}(z) \\
\lim _{m=\infty} \frac{S(z+m)}{\left(\mid \underline{m}-\mathrm{I} m^{z}\right)^{\mu}}=0
\end{array}\right.
$$

entspricht ein solches Fundamentalsystem particulärer Integrale

$$
y_{1}, y_{2}, \ldots ; y_{\mu}
$$

der linearen homogenen Differentialgleichung $\mu^{\text {ter }}$ Ordnung

dass

$$
D_{x} x D_{x} \ldots x D_{x} y=(-\mathrm{I})^{\prime} y,
$$

$$
S_{\lambda}(z)=\int_{0} y_{\lambda} x^{z-1} d x, \quad(\lambda=1,2, \ldots, \mu)
$$


Hj. Mellin.

wenn der reelle Theil von z positiv ist, und ungekehrt entspricht auch jedem Fundamentalsystem (67) ein Fundamentalsystem (66), fü welches die Gleichungen (68) stattinden.

Dieser Satz ist nur ein Specialfall von anderen, die sich auf lineare homogene Differentialgleichungen der Form

$$
x^{\alpha} D_{x} x^{\alpha_{1}} D_{x} \ldots D_{x} x^{\alpha_{m} y}=c x^{\beta} D_{x} x^{\beta_{1}} D_{x} \ldots D_{x} x^{\beta_{\nu}} y
$$

beziehen. Die Differentialgleichung der hypergeometrischen Reihe ist von der Form (69). Sie kann in der That folgendermaassen geschrieben werden:

$$
x^{\beta} D_{x} x^{\check{\gamma}} D_{x} y=x^{\check{\gamma}} D_{x} x^{\beta-\alpha+1} D_{x} x^{\alpha} y .
$$

Die Gammafunction ist nicht die einzige Function, welche nach der im Vorigen angewandten Methode behandelt werden kann und von der man alsdann zu neuen mit der Function verwandten Transcendenten gelangt. Dieselbe Methode kann ebenfalls auf die Function

$$
F(z)=\frac{\prod_{n=0}^{\infty}\left(\mathrm{I}+a_{1} q^{n} z\right)^{n_{1}} \ldots \prod_{n=0}^{\infty}\left(\mathrm{I}+a_{v} q^{n} z\right)^{u_{v_{n}}}}{\prod_{n=0}^{\infty}\left(\mathrm{I}+b_{1} q^{n} z\right)^{\nu_{1}} \ldots \prod_{n=0}^{\infty}\left(\mathrm{I}+b_{s} q^{n} z\right)^{\nu_{s}}} \quad(|q|<\mathrm{I})
$$

angewandt werden. 